\title{
Pacific
}

Journal of

Mathematics

\section{ON INTRINSICALLY KNOTTED OR COMPLETELY 3-LINKED GRAPHS}

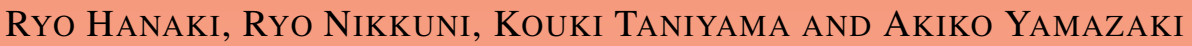




\title{
ON INTRINSICALLY KNOTTED OR COMPLETELY 3-LINKED GRAPHS
}

\author{
Ryo Hanaki, Ryo NikkUni, Kouki Taniyama And Akiko Yamazaki
}

\begin{abstract}
We say that a graph is intrinsically knotted or completely 3-linked if every embedding of the graph into the 3 -sphere contains a nontrivial knot or a 3-component link each of whose 2-component sublinks is nonsplittable. We show that a graph obtained from the complete graph on seven vertices by a finite sequence of $\Delta \mathrm{Y}$-exchanges and $\mathrm{Y} \Delta$-exchanges is a minor-minimal intrinsically knotted or completely 3 -linked graph.
\end{abstract}

\section{Introduction}

Throughout this paper we work in the piecewise linear category. Let $f$ be an embedding of a finite graph $G$ into the 3-sphere. Then $f$ is called a spatial embedding of $G$ and $f(G)$ is called a spatial graph. We denote the set of all spatial embeddings of $G$ by $\operatorname{SE}(G)$. We call a subgraph $\gamma$ of $G$ that is homeomorphic to the circle a cycle of $G$. For a positive integer $n$, let $\Gamma^{(n)}(G)$ denote the set of all cycles of $G$ if $n=1$ and the set of all unions of $n$ mutually disjoint cycles of $G$ if $n \geq 2$. For simplicity, we also write $\Gamma(G)$ for $\Gamma^{(1)}(G)$. For an element $\lambda$ in $\Gamma^{(n)}(G)$ and a spatial embedding $f$ of $G, f(\lambda)$ is a knot if $n=1$ and an $n$-component link if $n \geq 2$.

A graph $G$ is said to be intrinsically linked (IL) if for every spatial embedding $f$ of $G, f(G)$ contains a nonsplittable 2-component link. Conway and Gordon [1983] and Sachs [1984] showed that $K_{6}$ is IL, where $K_{m}$ denotes the complete graph on $m$ vertices. Also, IL graphs have been completely characterized as follows. For a graph $G$ and an edge $e$ of $G$, we denote the subgraph $G \backslash$ int $e$ by $G-e$. Let $e=\overline{u v}$ be an edge of $G$ that is not a loop. We call the graph obtained from $G-e$ by identifying the end vertices $u$ and $v$ the edge contraction of $G$ along $e$, and denote it by $G / e$. A graph $H$ is called a minor of a graph $G$ if there exists a subgraph $G^{\prime}$ of $G$ and edges $e_{1}, e_{2}, \ldots, e_{m}$ of $G^{\prime}$ such that $H$ is obtained from $G^{\prime}$ by a

Nikkuni was partially supported by Grant-in-Aid for Young Scientists (B) (No. 21740046), Japan Society for the Promotion of Science. Taniyama was partially supported by Grant-in-Aid for Scientific Research (C) (No. 21540099), Japan Society for the Promotion of Science.

MSC2000: primary 57M15; secondary 57M25.

Keywords: spatial graph, intrinsic knottedness, $\triangle \mathrm{Y}$-exchange, $\mathrm{Y} \triangle$-exchange. 
sequence of edge contractions along $e_{1}, e_{2}, \ldots, e_{m}$. A minor $H$ of $G$ is called a proper minor if $H$ does not equal $G$. Let $\mathscr{P}$ be a property for graphs that is closed under minor reductions; that is, for any graph $G$ that does not have $\mathscr{P}$, all minors of $G$ also do not have $\mathscr{P}$. A graph $G$ is said to be minor-minimal with respect to $\mathscr{P}$ if $G$ has $\mathscr{P}$ but all proper minors of $G$ do not have $\mathscr{P}$. Note that $G$ has $\mathscr{P}$ if and only if $G$ has a minor-minimal graph with respect to $\mathscr{P}$ as a minor. By the famous theorem of Robertson and Seymour [2004], there are finitely many minor-minimal graphs with respect to $\mathscr{P}$. Nešetrril and Thomas [1985] showed that IL is closed under minor reductions, and Robertson, Seymour and Thomas [Robertson et al. 1995] showed that the set of all minor-minimal graphs with respect to IL equals the Petersen family, which is the set of all graphs obtained from $K_{6}$ by a finite sequence of $\triangle \mathrm{Y}$-exchanges and $\mathrm{Y} \triangle$-exchanges. $\mathrm{A} \triangle \mathrm{Y}$-exchange is the left-to-right operation shown here:

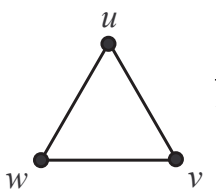

$G_{\triangle}$

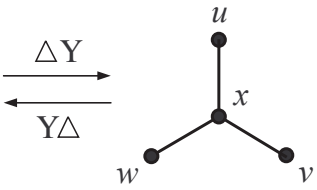

$G_{\mathrm{Y}}$

That is, a graph $G_{\triangle}$ containing a three-edge cycle $\triangle$ is changed into a new graph $G_{Y}$ by removing the edges of the cycle and adding a new vertex $x$ connected to each of the vertices of the deleted cycle, thus forming a Y. A Y $\triangle$-exchange is the reverse of this operation. $\triangle \mathrm{Y}$ - and $\mathrm{Y} \triangle$-exchanges preserve IL: if $G_{\triangle}$ is IL, so is $G_{\mathrm{Y}}$ [Motwani et al. 1988], and if $G_{\mathrm{Y}}$ is IL, so is $G_{\triangle}$ [Robertson et al. 1995].

The Petersen family contains seven graphs, including the Petersen graph $P_{10}$ :

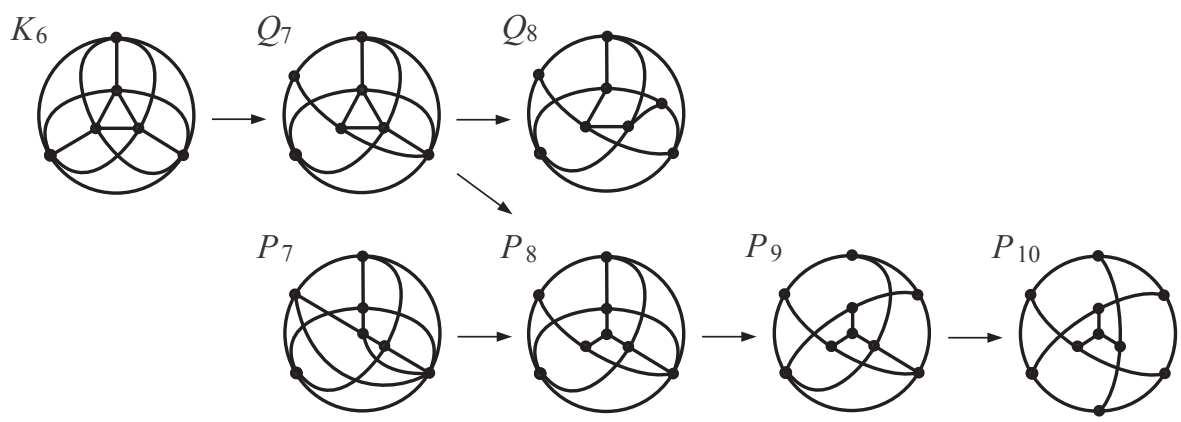

(An arrow between two graphs indicates the application of a single $\triangle \mathrm{Y}$-exchange.)

A graph $G$ is said to be intrinsically knotted (IK) if for every spatial embedding $f$ of $G, f(G)$ contains a nontrivial knot. Conway and Gordon [1983] showed that $K_{7}$ is IK. Fellows and Langston [1988] showed that IK is closed under minor 
reductions. Motwani, Raghunathan, and Saran [Motwani et al. 1988] showed that $K_{7}$ is a minor-minimal IK graph, and additional minor-minimal IK graphs were given in [Kohara and Suzuki 1992] and [Foisy 2002; 2003].

IK graphs have not been completely characterized yet. If $G_{\triangle}$ is IK then $G_{Y}$ is also IK [Motwani et al. 1988], but if $G_{Y}$ is IK then $G_{\triangle}$ may not always be IK. That is, the $\mathrm{Y} \triangle$-exchange does not preserve IK in general. Flapan and Naimi [2008] showed that there exists a graph $G_{F N}$ obtained from $K_{7}$ by five $\triangle \mathrm{Y}$-exchanges and two $\mathrm{Y} \triangle$-exchanges that is not IK. We call the set of all graphs obtained from $K_{7}$ by a finite sequence of $\triangle \mathrm{Y}$ and $\mathrm{Y} \triangle$-exchanges the Heawood family. ${ }^{1}$ This family contains exactly twenty graphs, as illustrated in Figure 1; of these, $C_{14}$ is the Heawood graph (Remark 4.7).

Kohara and Suzuki [1992] showed that a graph $G$ in the Heawood family is a minor-minimal IK graph if $G$ is obtained from $K_{7}$ by a finite sequence of $\Delta \mathrm{Y}$ exchanges, that is, if $G$ is one of fourteen graphs $K_{7}, H_{8}, H_{9}, \ldots, H_{12}, F_{9}, F_{10}$, $E_{10}, E_{11}$ and $C_{11}, C_{12}, \ldots, C_{14} .^{2}$ On the other hand, $N_{10}^{\prime}$ is isomorphic to $G_{F N}$, that is, $N_{10}^{\prime}$ is not IK. Our first purpose in this paper is to determine completely when a graph in the Heawood family is IK.

Theorem 1.1. For a graph $G$ in the Heawood family, the following are equivalent:

(1) $G$ is $I K$.

(2) $G$ is obtained from $K_{7}$ by a finite sequence of $\triangle \mathrm{Y}$-exchanges.

(3) $\Gamma^{(3)}(G)$ is the empty set.

Hence the members $N_{9}, N_{10}, N_{11}, N_{10}^{\prime}, N_{11}^{\prime}$ and $N_{12}^{\prime}$ of the Heawood family are not $I K$, and only they contain a union of three mutually disjoint cycles.

Our second purpose is to show that any of the graphs in the Heawood family is a minor-minimal graph with respect to a certain kind of intrinsic nontriviality even if it is not IK. We say that a graph $G$ is intrinsically knotted or completely 3-linked - $\mathrm{I}(\mathrm{K}$ or $\mathrm{C} 3 \mathrm{~L})$ for short - if for every spatial embedding $f$ of $G, f(G)$ contains a nontrivial knot or a 3-component link all of whose 2-component sublinks are nonsplittable. An IK graph is $\mathrm{I}(\mathrm{K}$ or $\mathrm{C} 3 \mathrm{~L})$. As we show in Proposition 2.2, I(K or $\mathrm{C} 3 \mathrm{~L}$ ) is closed under minor reductions.

Theorem 1.2. All graphs in the Heawood family are minor-minimal $I(K$ or $C 3 L)$ graphs.

As we have seen, $N_{9}, N_{10}, N_{11}, N_{10}^{\prime}, N_{11}^{\prime}$ and $N_{12}^{\prime}$ are not IK, but they are but $\mathrm{I}(\mathrm{K}$ or $\mathrm{C} 3 \mathrm{~L})$ and are minor-minimal with respect to $\mathrm{I}(\mathrm{K}$ or $\mathrm{C} 3 \mathrm{~L})$.

\footnotetext{
${ }^{1}$ Van der Holst [2006] calls the set of all graphs obtained from $K_{7}$ or $K_{3,3,1,1}$ by a finite sequence of $\Delta \mathrm{Y}$-exchanges and $\mathrm{Y} \triangle$-exchanges the Heawood family, where $K_{3,3,1,1}$ is the complete 4-partite graph on $3+3+1+1$ vertices.

${ }^{2}$ One edge of $F_{10}$ in [Kohara and Suzuki 1992, Figure 5] is wanting.
} 


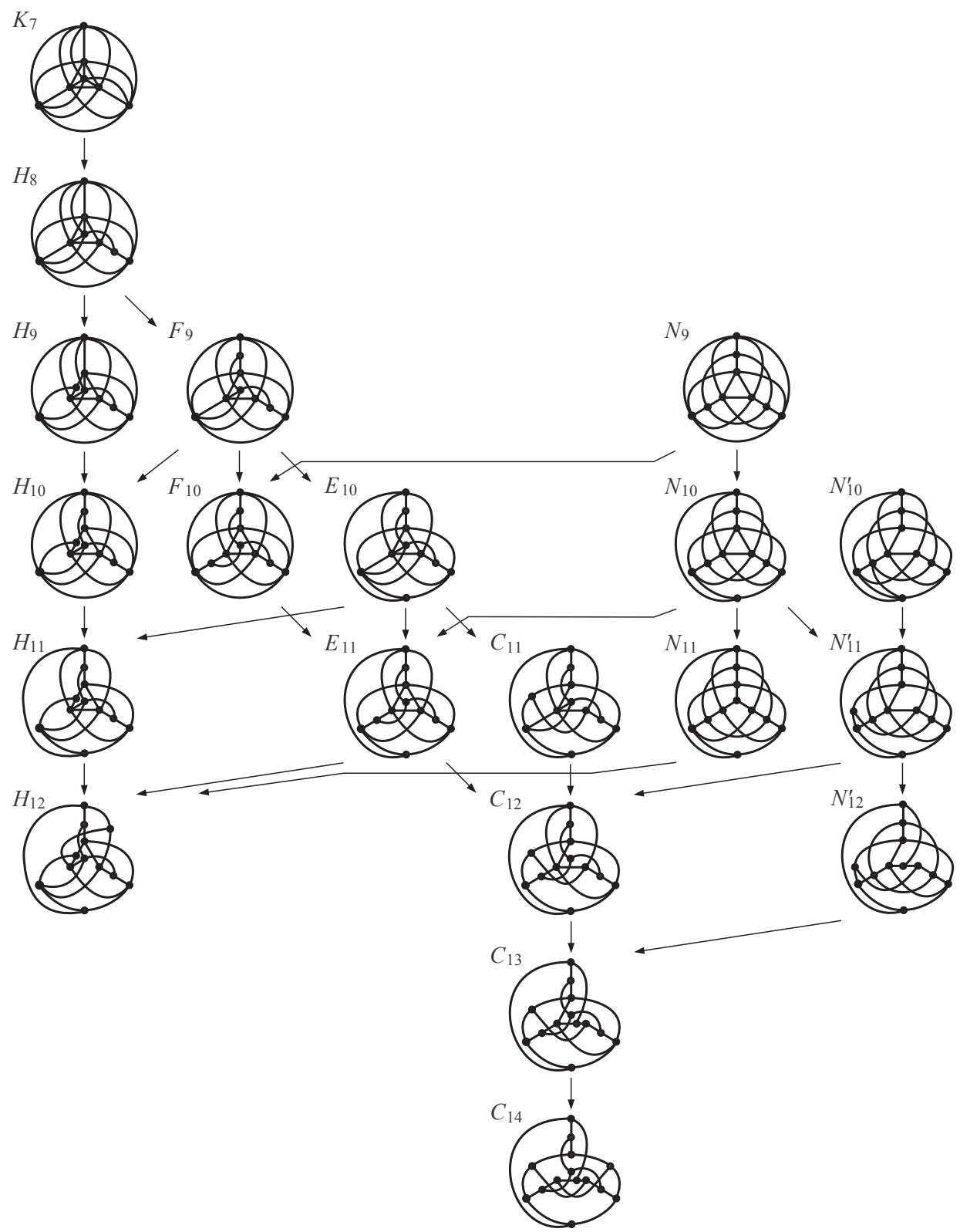

Figure 1. The Heawood family. An arrow between two graphs indicates the application of a single $\Delta \mathrm{Y}$-exchange.

Remark 1.3. A graph $G$ is said to be intrinsically $n$-linked $(\mathrm{I} n \mathrm{~L})$ if for every spatial embedding $f$ of $G, f(G)$ contains a nonsplittable $n$-component link [Flapan et al. 2001a; 2001b]. I2L coincides with IL. Let $G$ be a graph in the Heawood family 
that is not IK. Then we show in Example 4.6 that there exists a spatial embedding $f$ of $G$ such that $f(G)$ does not contain a nonsplittable 3-component link. That is, $G$ is neither IK nor I3L.

Remark 1.4. A graph $G$ is called intrinsically knotted or 3-linked- $\mathrm{I}(\mathrm{K}$ or $3 \mathrm{~L})$ for short - if for every spatial embedding $f$ of $G, f(G)$ contains a nontrivial knot or a nonsplittable 3-component link. Clearly $\mathrm{I}(\mathrm{K}$ or $\mathrm{C} 3 \mathrm{~L})$ implies $\mathrm{I}(\mathrm{K}$ or $3 \mathrm{~L})$, but the converse is not true: [Foisy 2006] exhibits an I(K or 3L) graph $G$ and a spatial embedding $f$ of $G$ such that $f(G)$ contains no nontrivial knot and all nonsplittable 3-component links contained in $f(G)$ have split 2-component sublinks.

The rest of this paper is organized as follows. Section 2 contains general results about graph minors, $\triangle \mathrm{Y}$-exchanges and spatial graphs. We prove Theorem $1.1 \mathrm{in}$ Section 3 and Theorem 1.2 in Section 4.

\section{Graph minors, $\Delta \mathrm{Y}$-exchanges and spatial graphs}

Let $H$ be a minor of a graph $G$. Then there exists a natural injection

$$
\Psi^{(n)}=\Psi_{H, G}^{(n)}: \Gamma^{(n)}(H) \longrightarrow \Gamma^{(n)}(G)
$$

for any positive integer $n$. We write $\Psi$ for $\Psi^{(1)}$. Let $f$ be a spatial embedding of $G$ and $e$ an edge of $G$ that is not a loop. Then by contracting $f(e)$ into one point, we obtain a spatial embedding $\psi(f)$ of $G / e$. Similarly, we can also obtain a spatial embedding $\psi(f)$ of $H$ from $f$. Thus we obtain a map

$$
\psi=\psi_{G, H}: \mathrm{SE}(G) \longrightarrow \mathrm{SE}(H) .
$$

Then we immediately have:

Proposition 2.1. For a spatial embedding $f$ of $G$ and an element $\lambda$ in $\Gamma^{(n)}(H)$, $\psi(f)(\lambda)$ is ambient isotopic to $f\left(\Psi^{(n)}(\lambda)\right)$.

Proposition 2.2. $I(K$ or $C 3 L)$ is closed under minor reductions.

Proof. Let $G$ be a graph that is not $\mathrm{I}(\mathrm{K}$ or $\mathrm{C} 3 \mathrm{~L})$, and $H$ be a minor of $G$. Let $f$ be a spatial embedding of $G$ that contains neither a nontrivial knot nor a 3-component link all of whose 2-component sublinks are nonsplittable. Then by Proposition 2.1, $\psi(f)$ has the same property. This implies that $H$ is not $\mathrm{I}(\mathrm{K}$ or $\mathrm{C} 3 \mathrm{~L})$.

Remark 2.3. Proposition 2.1 also implies that $\mathrm{IK}, \mathrm{I} n \mathrm{~L}$ and $\mathrm{I}(\mathrm{K}$ or $3 \mathrm{~L})$ are closed under minor reductions.

Let $G_{\triangle}$ and $G_{Y}$ be two graphs such that $G_{Y}$ is obtained from $G_{\triangle}$ by a single $\triangle \mathrm{Y}$-exchange, as in the previous section. Let $\lambda$ be an element in $\Gamma^{(n)}\left(G_{\triangle}\right)$ that does not contain $\triangle$. Then there exists an element $\Phi^{(n)}(\lambda)$ in $\Gamma^{(n)}\left(G_{Y}\right)$ such that 
$\lambda \backslash \triangle=\Phi^{(n)}(\lambda) \backslash \mathrm{Y}$. Thus we obtain a map

$$
\Phi^{(n)}=\Phi_{G_{\triangle}, G_{Y}}^{(n)}:\left\{\lambda \in \Gamma^{(n)}\left(G_{\triangle}\right) \mid \lambda \not \supset \triangle\right\} \longrightarrow \Gamma^{(n)}\left(G_{Y}\right),
$$

for any positive integer $n$. We denote $\Phi^{(1)}$ by $\Phi$. Note that $\Phi^{(n)}$ is surjective and the inverse image of $\lambda$ by $\Phi^{(n)}$ contains at most two elements in $\Gamma^{(n)}\left(G_{\triangle}\right)$ for any element $\lambda$ in $\Gamma^{(n)}\left(G_{Y}\right)$. The surjectivity of $\Phi^{(n)}$ implies Proposition 2.4.

Proposition 2.4. For $n \geq 2$, if $\Gamma^{(n)}\left(G_{\triangle}\right)=\varnothing$, then $\Gamma^{(n)}\left(G_{Y}\right)=\varnothing$.

Let $f$ be a spatial embedding of $G_{\mathrm{Y}}$, and let $D$ be a 2-disk in the 3-sphere such that $D \cap f\left(G_{\mathrm{Y}}\right)=f(\mathrm{Y})$ and $\partial D \cap f\left(G_{\mathrm{Y}}\right)=\{f(u), f(v), f(w)\}$. (Throughout the paper we use $u, v, w, x$ for the vertices of the $\mathrm{Y}$ of interest, as in the first figure on page 408), Let $\varphi(f)$ be a spatial embedding of $G_{\triangle}$ such that $\varphi(f)(x)=f(x)$ for $x \in G_{\mathrm{Y}} \backslash \mathrm{Y}$ and $\varphi(f)\left(G_{\triangle}\right)=\left(f\left(G_{\mathrm{Y}}\right) \backslash f(\mathrm{Y})\right) \cup \partial D$. Then we obtain a map

$$
\varphi=\varphi_{G_{\mathrm{Y}}, G_{\triangle}}: \operatorname{SE}\left(G_{\mathrm{Y}}\right) \longrightarrow \mathrm{SE}\left(G_{\triangle}\right),
$$

and we immediately have Proposition 2.5.

Proposition 2.5. For a spatial embedding $f$ of $G_{\mathrm{Y}}$ and an element $\lambda$ in $\Gamma^{(n)}\left(G_{\mathrm{Y}}\right)$, $f(\lambda)$ is ambient isotopic to $\varphi(f)\left(\lambda^{\prime}\right)$ for each element $\lambda^{\prime}$ in the inverse image of $\lambda$ by $\Phi^{(n)}$.

Lemma 2.6. If $G_{\triangle}$ is $I(K$ or $C 3 L)$, then $G_{Y}$ is also $I(K$ or $C 3 L)$.

Proof. Assume that $G_{\mathrm{Y}}$ is not $\mathrm{I}(\mathrm{K}$ or $\mathrm{C} 3 \mathrm{~L})$, that is, that there exists a spatial embedding $f$ of $G_{\mathrm{Y}}$ that contains neither a nontrivial knot nor a 3-component link all of whose 2-component sublinks are nonsplittable. We show that $\varphi(f)\left(G_{\triangle}\right)$ also has the same property.

Let $\gamma$ be an element in $\Gamma\left(G_{\triangle}\right)$. If $\gamma$ is not $\triangle$, then $\varphi(f)(\gamma)$ is ambient isotopic to $f(\Phi(\gamma))$ by Proposition 2.5, and $f(\Phi(\gamma))$ is a trivial knot by the assumption. Since $\varphi(f)(\triangle)$ is also a trivial knot, it follows that $\varphi(f)\left(G_{\triangle}\right)$ does not contain a nontrivial knot. Let $\lambda$ be an element in $\Gamma^{(3)}\left(G_{\triangle}\right)$. If $\lambda$ does not contain $\Delta$, then $\varphi(f)(\lambda)$ is ambient isotopic to $f\left(\Phi^{(3)}(\lambda)\right)$ by Proposition 2.5, and $f\left(\Phi^{(3)}(\lambda)\right)$ is a 3 -component link that contains a split 2-component sublink by the assumption. If $\lambda$ contains $\triangle$, then $\varphi(f)(\lambda)$ is a split 3-component link. Thus we see that $\varphi(f)\left(G_{\triangle}\right)$ does not contain a 3-component link with a nonsplittable 2-component sublink.

Lemma 2.7. If $G_{Y}$ is minor-minimal for $I(K$ or $C 3 L)$, then $G_{\triangle}$ is also minorminimal for $I(K$ or $C 3 L)$.

Proof. (This lemma has already been proven in more general form [Ozawa and Tsutsumi 2007, Lemma 3.1, Exercise 3.2], but we prove it here for convenience.) We show that for any edge $e$ of $G_{\triangle}$ that is not a loop, there exist a spatial embedding $f$ of $G_{\triangle}-e$ and a spatial embedding $g$ of $G_{\triangle} / e$ such that each of $f\left(G_{\triangle}-e\right)$ and 
$g\left(G_{\triangle} / e\right)$ contains neither a nontrivial knot nor a 3-component link all of whose 2-component sublink are nonsplittable. If $e$ is not one of the edges $\overline{u v}, \overline{v w}$ or $\overline{w u}$ of the $\triangle$ then there exist a spatial embedding $f^{\prime}$ of $G_{Y}-e$ and a spatial embedding $g^{\prime}$ of $G_{\mathrm{Y}} / e$ such that both $f^{\prime}\left(G_{\mathrm{Y}}-e\right)$ and $g^{\prime}\left(G_{\mathrm{Y}} / e\right)$ contain neither a nontrivial knot nor a 3-component link all of whose 2-component sublinks are nonsplittable. The graph $G_{\mathrm{Y}}-e$ is obtained from $G_{\triangle}-e$, and likewise $G_{\mathrm{Y}} / e$ from $G_{\triangle} / e$, by a single $\triangle \mathrm{Y}$-exchange at the same $\triangle$. Then we see that each of $\varphi\left(f^{\prime}\right)\left(G_{\triangle}-e\right)$ and $\varphi\left(g^{\prime}\right)\left(G_{\triangle} / e\right)$ contains neither a nontrivial knot nor a 3-component link having only nonsplittable 2-component sublinks, in a way similar to the proof of Lemma 2.6. If $e$ is one of $\overline{u v}, \overline{v w}$ and $\overline{w u}$, we may assume that $e=\overline{u v}$ without loss of generality. Now there exists a spatial embedding $f^{\prime}$ of $G_{Y} / \overline{x w}$ such that $f^{\prime}\left(G_{Y} / \overline{x w}\right)$ contains neither a nontrivial knot nor a 3-component link having only nonsplittable 2-component sublinks. Then we can see that $G_{\triangle}-\overline{u v}=G_{\mathrm{Y}} / \overline{x w}$. On the other hand, there exists a spatial embedding $g^{\prime}$ of $G_{Y} / \overline{x v} / \overline{x u}$ such that $g^{\prime}\left(G_{Y} / \overline{x v} / \overline{x u}\right)$ contains neither a nontrivial knot nor a 3-component link having only nonsplittable 2-component sublink. Take a 2-disk $D^{\prime}$ in the 3-sphere such that $D^{\prime} \cap g^{\prime}\left(G_{\mathrm{Y}} / \overline{x v} / \overline{x u}\right)=g^{\prime}(\overline{u w})$ and $\partial D^{\prime} \cap g^{\prime}\left(G_{\mathrm{Y}} / \overline{x v} / \overline{x u}\right)=\left\{g^{\prime}(u), g^{\prime}(w)\right\}$. Then $\left(g^{\prime}\left(G_{Y} / \overline{x v} / \overline{x u}\right) \backslash \operatorname{int} g^{\prime}(\overline{u w})\right) \cup \partial D^{\prime}$ may be regarded as the image of a spatial embedding of $G_{\triangle} / \overline{u v}$, denoted by $g$. Clearly $g\left(G_{\triangle} / \overline{u v}\right)$ contains neither a nontrivial knot nor a 3-component link having only nonsplittable 2-component sublink.

\section{Proof of Theorem 1.1}

Lemma 3.1. Each of the graphs $N_{9}, N_{10}, N_{11}, N_{10}^{\prime}, N_{11}^{\prime}$ and $N_{12}^{\prime}$ in the Heawood family is not IK.

Proof. For $N_{10}^{\prime}$, see [Flapan and Naimi 2008]. We show that $N_{9}, N_{10}, N_{11}, N_{11}^{\prime}$ and $N_{12}^{\prime}$ are not IK. Let $f_{9}$ be the spatial embedding of $N_{9}$ illustrated in Figure 2 . It can be checked directly that $f_{9}\left(N_{9}\right)$ does not contain a nontrivial knot. Thus $N_{9}$ is

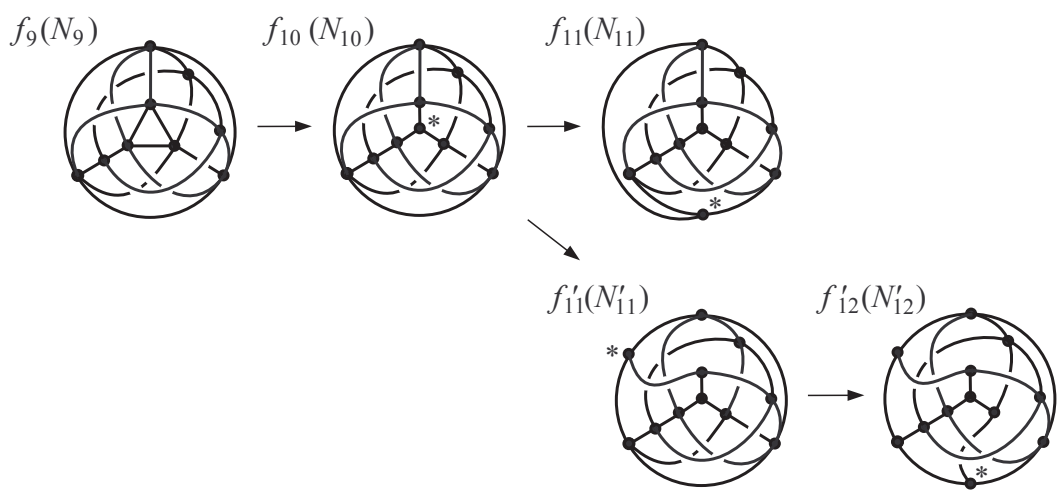

Figure 2 
not IK. Let $f_{10}$ be the spatial embedding of $N_{10}$ illustrated in Figure 2. Let $\varphi_{N_{10}, N_{9}}$ be the map from $\operatorname{SE}\left(N_{10}\right)$ to $\operatorname{SE}\left(N_{9}\right)$ induced by the $\mathrm{Y} \triangle$-exchange from $N_{10}$ to $N_{9}$ at the Y-fork marked $*$ in Figure 2 . Then clearly $\varphi\left(f_{10}\right)=f_{9}$. Since $f_{9}\left(N_{9}\right)$ does not contain a nontrivial knot, by Proposition 2.5 it follows that $f_{10}\left(N_{10}\right)$ also does not contain a nontrivial knot. Thus, $N_{10}$ is not IK. By repeating this argument, we can see that each of the graphs $N_{11}, N_{11}^{\prime}$ and $N_{12}^{\prime}$ is also not IK; see Figure 2.

Proof of Theorem 1.1. First we show that (1) and (2) are equivalent. Since we already know that (2) implies (1), we show that (1) implies (2). If $G$ is IK, then by Lemma 3.1 we see that $G$ is not one of $N_{9}, N_{10}, N_{11}, N_{10}^{\prime}, N_{11}^{\prime}$ or $N_{12}^{\prime}$. Thus $G$ is obtained from $K_{7}$ by a finite sequence of $\triangle \mathrm{Y}$-exchanges. Next we show that (2) and (3) are equivalent. Assume that $G$ is obtained from $K_{7}$ by a finite sequence of $\triangle$ Y-exchanges. $\Gamma^{(3)}\left(K_{7}\right)$ is the empty set. Thus, by Proposition 2.4 , we see that $\Gamma^{(3)}(G)$ is the empty set. Conversely, if $G$ is one of $N_{9}, N_{10}, N_{11}, N_{10}^{\prime}, N_{11}^{\prime}$, and $N_{12}^{\prime}$, then $\Gamma^{(3)}(G)$ is not the empty set. This completes the proof.

Remark 3.2. Let $f_{11}^{\prime}$ be the spatial embedding of $N_{11}^{\prime}$ illustrated in Figure 2, and let $f_{10}^{\prime}$ be the spatial embedding of $N_{10}^{\prime}$ illustrated in the figure below. Let $\varphi_{N_{11}^{\prime}, N_{10}^{\prime}}$ be the map from $\operatorname{SE}\left(N_{11}^{\prime}\right)$ to $\operatorname{SE}\left(N_{10}^{\prime}\right)$ induced by the $\mathrm{Y} \triangle$-exchange from $N_{11}^{\prime}$ to $N_{10}^{\prime}$ at the Y-fork marked $* *$. Then clearly $\varphi\left(f_{11}^{\prime}\right)=f_{10}^{\prime}$. Also, we can see that $f_{10}^{\prime}$ coincides with Flapan and Naimi's example [2008] of a spatial embedding of $N_{10}^{\prime}$ whose image does not contain a nontrivial knot, as illustrated in the leftmost diagram:
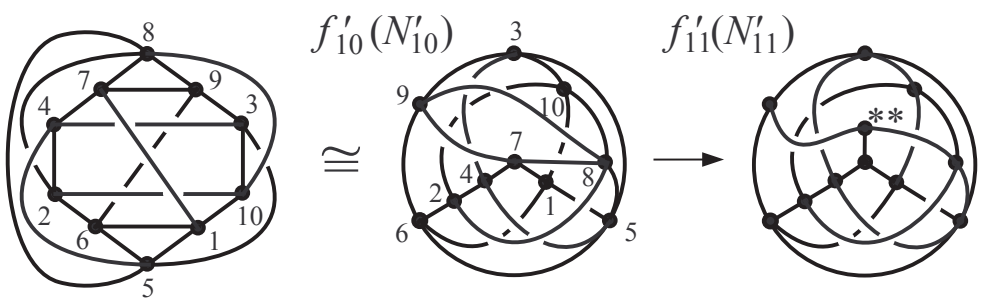

\section{Proof of Theorem 1.2}

Lemma 4.1 [Conway and Gordon 1983; Taniyama and Yasuhara 2001]. Let G be a graph in the Petersen family and $f$ a spatial embedding of $G$. Then there exists an element $\lambda$ in $\Gamma^{(2)}(G)$ such that $\operatorname{lk}(f(\lambda))$ is odd, where $1 \mathrm{k}$ denotes the linking number in the 3-sphere.

Let $D_{4}$ be the graph illustrated on the right. We denote the set of all cycles of $D_{4}$ with exactly four edges by $\Gamma_{4}\left(D_{4}\right)$. For a spatial embedding $f$ of $D_{4}$, we define

$$
\alpha(f) \equiv \sum_{\gamma \in \Gamma_{4}\left(D_{4}\right)} a_{2}(f(\gamma))(\bmod 2),
$$

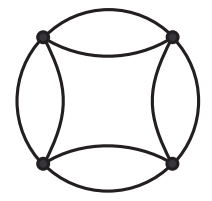

$D_{4}$ 
where $a_{2}$ denotes the second coefficient of the Conway polynomial. Note that $a_{2}(K)$ of a knot $K$ is congruent to the Arf invariant modulo 2 [Kauffman 1983].

Lemma 4.2 [Taniyama and Yasuhara 2001]. Let $f$ be a spatial embedding of $D_{4}$ and $\lambda, \lambda^{\prime}$ all elements in $\Gamma^{(2)}\left(D_{4}\right)$. If both $\operatorname{lk}(f(\lambda))$ and $\operatorname{lk}\left(f\left(\lambda^{\prime}\right)\right)$ are odd, then $\alpha(f)=1$.

Let $G$ be a graph that contains $D_{4}$ as a minor and $f$ a spatial embedding of $G$. Then we define

$$
\alpha(f) \equiv \sum_{\gamma \in \Gamma_{4}\left(D_{4}\right)} a_{2}\left(f\left(\Psi_{D_{4}, G}(\gamma)\right)\right)(\bmod 2) .
$$

Lemma 4.3. Let $G$ be a graph that contains $D_{4}$ as a minor and let $f$ be a spatial embedding of $G$. For two elements $\mu$ and $\mu^{\prime}$ in $\Psi_{D_{4}, G}^{(2)}\left(\Gamma^{(2)}\left(D_{4}\right)\right)$, if both $\operatorname{lk}(f(\mu))$ and $\operatorname{lk}\left(f\left(\mu^{\prime}\right)\right)$ are odd, then $\alpha(f)=1$.

Proof. For two elements $\lambda$ and $\lambda^{\prime}$ in $\Gamma^{(2)}\left(D_{4}\right)$, we see that both $\operatorname{lk}\left(f\left(\Psi_{D_{4}, G}^{(2)}(\lambda)\right)\right)$ and $\operatorname{lk}\left(f\left(\Psi_{D_{4}, G}^{(2)}\left(\lambda^{\prime}\right)\right)\right)$ are odd by the assumption. Then by Proposition 2.1 , it follows that $\operatorname{lk}\left(\psi_{G, D_{4}}(f)(\lambda)\right)$ and $\operatorname{lk}\left(\psi_{G, D_{4}}(f)\left(\lambda^{\prime}\right)\right)$ are also odd. Therefore, by Lemma 4.2, we have that

$$
\alpha(f) \equiv \sum_{\gamma \in \Gamma_{4}\left(D_{4}\right)} a_{2}\left(f\left(\Psi_{D_{4}, G}(\gamma)\right)\right)=\sum_{\gamma \in \Gamma_{4}\left(D_{4}\right)} a_{2}\left(\psi_{G, D_{4}}(f)(\gamma)\right) \equiv 1(\bmod 2) .
$$

The next theorem is the most important part of the proof of Theorem 1.2.

Theorem 4.4. Let $G$ be $N_{9}$ or $N_{10}^{\prime}$. For every spatial embedding $f$ of $G$, there exists an element $\gamma$ in $\Gamma(G)$ such that $a_{2}(f(\gamma))$ is odd, or there exists an element $\lambda$ in $\Gamma^{(3)}(G)$ such that each 2-component sublink of $f(\lambda)$ has an odd linking number. Proof. We will denote by $\left[i_{1} i_{2} \ldots i_{k}\right]$ the cycle $\overline{i_{1} i_{2}} \cup \overline{i_{2} i_{3}} \cup \ldots \cup \overline{i_{k-1} i_{k}} \cup \overline{i_{k} i_{1}}$ of $G$. We label each vertex of $G$ as follows:
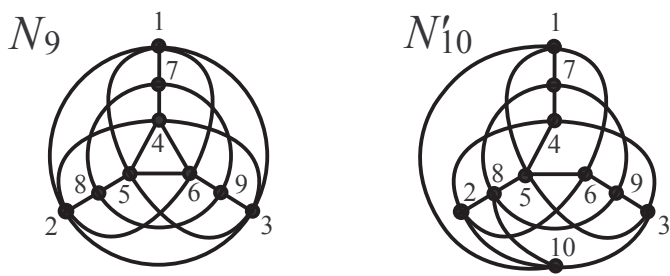

First we show the case of $G=N_{9}$. Let $f$ be a spatial embedding of $N_{9}$. Note that $N_{9}$ contains $K_{6}$ as the proper minor

$$
\left(\left(\left(N_{9}-\overline{78}\right)-\overline{89}\right)-\overline{97}\right) / \overline{47} / \overline{58} / \overline{69} .
$$

By Lemma 4.1, there is thus an element $v$ in $\Gamma^{(2)}\left(K_{6}\right)$ such that $\operatorname{lk}\left(\psi_{N_{9}, K_{6}}(f)(v)\right)$ is odd. Hence, by Proposition 2.1, there exists an element $\mu$ in $\Psi_{K_{6}, N_{9}}^{(2)}\left(\Gamma^{(2)}\left(K_{6}\right)\right)$ such that $\operatorname{lk}(f(\mu))$ is odd. $\Psi_{K_{6}, N_{9}}^{(2)}\left(\Gamma^{(2)}\left(K_{6}\right)\right)$ consists of ten elements, and by the 
symmetry of $N_{9}$, we may assume that $\mu=\left[\begin{array}{lll}174 & 4\end{array}\right] \cup[2658]$ or [ 1123$] \cup[456]$ without loss of generality.

Case 1. Let $\mu=[1743] \cup[2658]$. Note that $N_{9}$ contains $P_{7}$ as the proper minor

$$
\left.\left(\left(\left(\left(N_{9}-\overline{61}\right)-\overline{62}\right)-\overline{64}\right)-\overline{65}\right)-\overline{69}\right) / \overline{39} .
$$

Thus, by Lemma 4.1, there is an element $v^{\prime}$ in $\Gamma^{(2)}\left(P_{7}\right)$ such that $\operatorname{lk}\left(\psi_{N_{9}, P_{7}}(f)\left(v^{\prime}\right)\right)$ is odd. Hence, by Proposition 2.1, there exists an element $\mu^{\prime}$ in $\Psi_{P_{7}, N_{9}}^{(2)}\left(\Gamma^{(2)}\left(P_{7}\right)\right)$ such that $\operatorname{lk}\left(f\left(\mu^{\prime}\right)\right)$ is odd. $\Psi_{P_{7}, N_{9}}^{(2)}\left(\Gamma^{(2)}\left(P_{7}\right)\right)$ consists of the nine elements

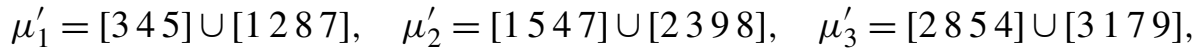

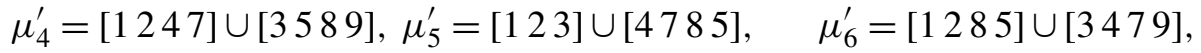

$$
\begin{aligned}
& \mu_{7}^{\prime}=[234] \cup[1587], \quad \mu_{8}^{\prime}=\left[\begin{array}{ll}
789 \\
\hline
\end{array} \cup[1245], \quad \mu_{9}^{\prime}=[153] \cup[2874]\right. \text {. }
\end{aligned}
$$

For $i=1,2, \ldots, 9$, let $J^{i}$ be the subgraph of $N_{9}$ that is $\mu \cup \mu_{i}^{\prime} \cup \overline{69}$ if $i=3,6$ and $\mu \cup \mu_{i}^{\prime}$ if $i \neq 3,6$. Assume that $\operatorname{lk}\left(f\left(\mu_{i}^{\prime}\right)\right)$ is odd for some $i \neq 8$. Then it can be easily seen that $J^{i}$ contains a graph $D^{i}$ as a minor, such that $D^{i}$ is isomorphic to $D_{4}$ and $\left\{\mu, \mu_{i}^{\prime}\right\}=\Psi_{D^{i}, J^{i}}^{(2)}\left(\Gamma^{(2)}\left(D^{i}\right)\right)$. Since both $\operatorname{lk}(f(\mu))$ and $\operatorname{lk}\left(f\left(\mu_{i}^{\prime}\right)\right)$ are odd, by Lemma 4.3 there exists an element $\gamma$ in $\Gamma\left(J^{i}\right)$ such that $a_{2}(f(\gamma))$ is odd. Next assume that $\operatorname{lk}\left(f\left(\mu_{8}^{\prime}\right)\right)$ is odd. We denote two elements [7 89] $\cup[1265$ ] and [7 8 9] $\cup\left[\begin{array}{ll}42 & 65\end{array}\right]$ in $\Gamma^{(2)}\left(J^{8}\right)$ by $\mu_{8,1}^{\prime}$ and $\mu_{8,2}^{\prime}$, respectively. We denote the subgraph $\mu \cup \mu_{8, j}^{\prime}$ of $J^{8}$ by $J^{8, j}(j=1,2)$. Then it can be easily seen that $J^{8, j}$ contains a graph $D^{8, j}$ as a minor, such that $D^{8, j}$ is isomorphic to $D_{4}$ and $\left\{\mu, \mu_{8, j}^{\prime}\right\}=$ $\Psi_{D^{8, j}, J^{8, j}}^{(2)}\left(\Gamma^{(2)}\left(D^{8, j}\right)\right)(j=1,2)$. Note that

$$
\left[\begin{array}{llll}
1 & 2 & 4 & 5
\end{array}\right]=\left[\begin{array}{llll}
1 & 2 & 6 & 5
\end{array}\right]+\left[\begin{array}{llll}
4 & 2 & 6 & 5
\end{array}\right]
$$

in $H_{1}\left(J^{8} ; \mathbb{Z}_{2}\right)$, where $H_{*}\left(\cdot ; \mathbb{Z}_{2}\right)$ denotes the homology group with $\mathbb{Z}_{2}$-coefficients. Then, by the homological property of the linking number, we have that

$$
1 \equiv \operatorname{lk}\left(f\left(\mu_{8}^{\prime}\right)\right) \equiv \operatorname{lk}\left(f\left(\mu_{8,1}^{\prime}\right)\right)+\operatorname{lk}\left(f\left(\mu_{8,2}^{\prime}\right)\right)(\bmod 2) .
$$

Thus we see that $\operatorname{lk}\left(f\left(\mu_{8,1}^{\prime}\right)\right)$ is odd or $\operatorname{lk}\left(f\left(\mu_{8,2}^{\prime}\right)\right)$ is odd. In either case, by Lemma 4.3 there exists an element $\gamma$ in $\Gamma\left(J^{8, j}\right)$ such that $a_{2}(f(\gamma))$ is odd.

Case 2. Let $\mu=\left[\begin{array}{lll}1 & 2 & 3\end{array}\right] \cup\left[\begin{array}{ll}4 & 56\end{array}\right]$. Note that $N_{9}$ contains $P_{9}$ as the proper minor

$$
\left.\left(\left(\left(\left(N_{9}-\overline{12}\right)-\overline{23}\right)-\overline{31}\right)-\overline{45}\right)-\overline{56}\right)-\overline{64} \text {. }
$$

Thus, by Lemma 4.1, there is an element $v^{\prime}$ in $\Gamma^{(2)}\left(P_{9}\right)$ such that $\operatorname{lk}\left(\psi_{N_{9}, P_{9}}(f)\left(v^{\prime}\right)\right)$ is odd. Hence by Proposition 2.1, there exists an element $\mu^{\prime}$ in $\Psi_{P_{9}, N_{9}}^{(2)}\left(\Gamma^{(2)}\left(P_{9}\right)\right)$ such that $\operatorname{lk}\left(f\left(\mu^{\prime}\right)\right)$ is odd. $\Psi_{P_{9}, N_{9}}^{(2)}\left(\Gamma^{(2)}\left(P_{9}\right)\right)$ consists of seven elements, and by the

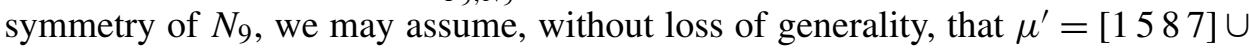
[26934] or [7 89] $\cup[153426]$. Denote by $J$ the subgraph $\mu \cup \mu^{\prime}$ of $N_{9}$. Assume 


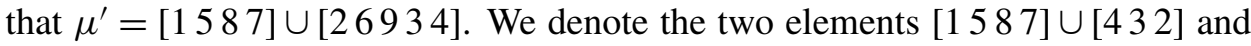
[1 58 8 7] $\cup\left[\begin{array}{ll}693 & 2\end{array}\right]$ in $\Gamma^{(2)}(J)$ by $\mu_{1}^{\prime}$ and $\mu_{2}^{\prime}$, respectively. We denote the subgraph $\mu \cup \mu_{i}^{\prime}$ of $J$ by $J^{i}(i=1,2)$. Then $J^{i}$ contains a graph $D^{i}$ as a minor such that $D^{i}$ is isomorphic to $D_{4}$ and

$$
\left\{\mu, \mu_{i}^{\prime}\right\}=\Psi_{D^{i}, J^{i}}^{(2)}\left(\Gamma^{(2)}\left(D^{i}\right)\right) \quad(i=1,2) .
$$

Since $[26934]=[432]+[6932]$ in $H_{1}\left(J ; \mathbb{Z}_{2}\right)$, it follows that

$$
1 \equiv \operatorname{lk}\left(f\left(\mu^{\prime}\right)\right) \equiv \operatorname{lk}\left(f\left(\mu_{1}^{\prime}\right)\right)+\operatorname{lk}\left(f\left(\mu_{2}^{\prime}\right)\right)(\bmod 2) .
$$

This implies that $\operatorname{lk}\left(f\left(\mu_{1}^{\prime}\right)\right)$ is odd or $\operatorname{lk}\left(f\left(\mu_{2}^{\prime}\right)\right)$ is odd. In both cases, by Lemma 4.3 there exists an element $\gamma$ in $\Gamma\left(J^{i}\right)$ such that $a_{2}(f(\gamma))$ is odd. Next assume that

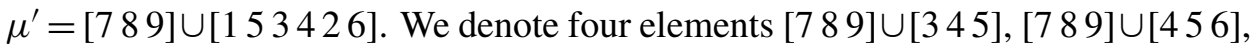
[7 89] $\cup[156]$ and [7 89] $\cup[246]$ in $\Gamma^{(2)}(J)$ by $\mu_{1}^{\prime}, \mu_{2}^{\prime}, \mu_{3}^{\prime}$ and $\mu_{4}^{\prime}$, respectively.

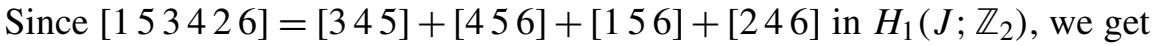

$$
1 \equiv \mathrm{lk}\left(\mu^{\prime}\right) \equiv \mathrm{lk}\left(\mu_{1}^{\prime}\right)+\operatorname{lk}\left(\mu_{2}^{\prime}\right)+\operatorname{lk}\left(\mu_{3}^{\prime}\right)+\operatorname{lk}\left(\mu_{4}^{\prime}\right)(\bmod 2) .
$$

This implies that $1 \mathrm{k}\left(\mu_{i}^{\prime}\right)$ is odd for some $i=1,2,3$ or 4 . Moreover, by the symmetry of $J$, we may assume that $\operatorname{lk}\left(\mu_{1}^{\prime}\right)$ is odd or $\operatorname{lk}\left(\mu_{2}^{\prime}\right)$ is odd without loss of generality. Assume that $\operatorname{lk}\left(\mu_{1}^{\prime}\right)$ is odd. We denote the subgraph $\mu \cup \mu_{1}^{\prime} \cup \overline{17} \cup \overline{69}$ of $N_{9}$ by $J^{1}$. Then $J^{1}$ contains a graph $D^{1}$ as a minor such that $D^{1}$ is isomorphic to $D_{4}$ and $\left\{\mu, \mu_{1}^{\prime}\right\}=\Psi_{D^{1}, J^{1}}^{(2)}\left(\Gamma^{(2)}\left(D^{1}\right)\right)$. Since both $\operatorname{lk}(f(\mu))$ and $\operatorname{lk}\left(f\left(\mu_{1}^{\prime}\right)\right)$ are odd, by Lemma 4.3 there exists an element $\gamma$ in $\Gamma\left(J^{1}\right)$ such that $a_{2}(f(\gamma))$ is odd. Next assume that $\operatorname{lk}\left(\mu_{2}^{\prime}\right)$ is odd. We denote four elements [7 8 9] $\cup\left[\begin{array}{ll}1 & 26\end{array}\right]$, [7 8 9] $\cup\left[\begin{array}{ll}1 & 23\end{array}\right]$, [7 8 9] $\cup[234]$ and [7 89] $\cup[135]$ in $\Gamma^{(2)}(J)$ by $\mu_{5}^{\prime}, \mu_{6}^{\prime}, \mu_{7}^{\prime}$ and $\mu_{8}^{\prime}$, respectively.

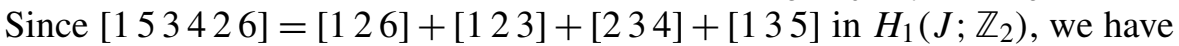

$$
1 \equiv \operatorname{lk}\left(\mu^{\prime}\right) \equiv \operatorname{lk}\left(\mu_{5}^{\prime}\right)+\operatorname{lk}\left(\mu_{6}^{\prime}\right)+\operatorname{lk}\left(\mu_{7}^{\prime}\right)+\operatorname{lk}\left(\mu_{8}^{\prime}\right)(\bmod 2) \text {. }
$$

Thus we see that $\operatorname{lk}\left(\mu_{i}^{\prime}\right)$ is odd for some $i=5,6,7$ or 8 . Moreover, by the symmetry of $J$, we may assume that $\operatorname{lk}\left(\mu_{5}^{\prime}\right)$ is odd or $\operatorname{lk}\left(\mu_{6}^{\prime}\right)$ is odd without loss of generality. Assume that $\operatorname{lk}\left(\mu_{5}^{\prime}\right)$ is odd. We denote the subgraph $\mu \cup \mu_{5}^{\prime} \cup \overline{47} \cup \overline{39}$ of $N_{9}$ by $J^{5}$. Then $J^{5}$ contains a graph $D^{5}$ as a minor such that $D^{5}$ is isomorphic to $D_{4}$ and $\left\{\mu, \mu_{5}^{\prime}\right\}=\Psi_{D^{5} J^{5}}^{(2)}\left(\Gamma^{(2)}\left(D^{5}\right)\right)$. Since both $\operatorname{lk}(f(\mu))$ and $\operatorname{lk}\left(f\left(\mu_{5}^{\prime}\right)\right)$ are odd, by Lemma 4.3 there exists an element $\gamma$ in $\Gamma\left(J^{5}\right)$ such that $a_{2}(f(\gamma))$ is odd. Finally, assume that $\operatorname{lk}\left(\mu_{6}^{\prime}\right)$ is odd. Let us consider the 3-component link $L=$

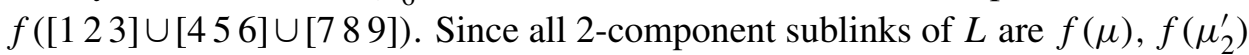
and $f\left(\mu_{6}^{\prime}\right)$, each of the 2-component sublinks of $L$ has an odd linking number.

Now we show the case of $G=N_{10}^{\prime}$. Let $f$ be a spatial embedding of $N_{10}^{\prime}$. Note that $N_{10}^{\prime}$ contains $P_{7}$ as the proper minor

$$
\left(\left(\left(N_{10}^{\prime}-\overline{78}\right)-\overline{89}\right)-\overline{97}\right) / \overline{47} / \overline{58} / \overline{69} .
$$


Thus by Lemma 4.1, there is an element $v$ in $\Gamma^{(2)}\left(P_{7}\right)$ such that $\operatorname{lk}\left(\psi_{N_{10}^{\prime}, P_{7}}(f)(v)\right)$ is odd. Hence by Proposition 2.1, there exists an element $\mu$ in $\Psi_{P_{7}, N_{10}^{\prime}}^{(2)}\left(\Gamma^{(2)}\left(P_{7}\right)\right)$ such that $\operatorname{lk}(f(\mu))$ is odd. $\Psi_{P_{7}, N_{10}^{\prime}}^{(2)}\left(\Gamma^{(2)}\left(P_{7}\right)\right)$ consists of nine elements, and by the symmetry of $N_{10}^{\prime}$, we may assume that $\mu=[1745] \cup[210396]$, [2 458$] \cup$

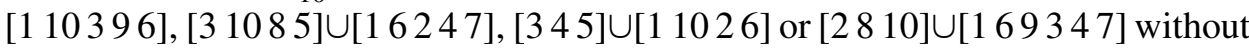
loss of generality.

Case 1. Let $\mu=[1745] \cup[210396]$. Note that $N_{10}^{\prime}$ contains $P_{9}$ as the proper minor

$$
\left.\left(\left(\left(\left(N_{10}^{\prime}-\overline{51}\right)-\overline{53}\right)-\overline{54}\right)-\overline{56}\right)-\overline{58}\right)-\overline{79} .
$$

Thus by Lemma 4.1 , there is an element $v^{\prime}$ in $\Gamma^{(2)}\left(P_{9}\right)$ such that $\operatorname{lk}\left(\psi_{N_{10}^{\prime}, P_{9}}(f)\left(v^{\prime}\right)\right)$ is odd. Hence by Proposition 2.1, there exists an element $\mu^{\prime}$ in $\Psi_{P_{9}, N_{10}^{\prime}}^{(2)}\left(\Gamma^{(2)}\left(P_{9}\right)\right)$ such that $\operatorname{lk}\left(f\left(\mu^{\prime}\right)\right)$ is odd. $\Psi_{P_{9}, N_{10}^{\prime}}^{(2)}\left(\Gamma^{(2)}\left(P_{9}\right)\right)$ consists of seven elements

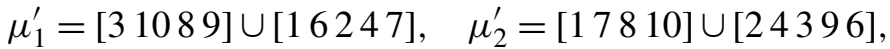

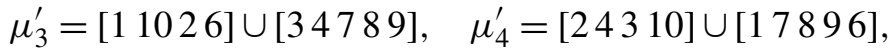

$$
\begin{aligned}
& \mu_{5}^{\prime}=\left[\begin{array}{lll}
24 & 48
\end{array}\right] \cup\left[\begin{array}{llll}
1 & 10396
\end{array}, \quad \mu_{6}^{\prime}=\left[\begin{array}{lll}
2 & 896
\end{array}\right] \cup\left[\begin{array}{lll}
1 & 10347
\end{array}\right]\right. \text {, }
\end{aligned}
$$

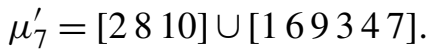

For $i=1,2, \ldots, 7$, let $J^{i}$ be the subgraph of $N_{10}^{\prime}$ that is $\mu \cup \mu_{i}^{\prime} \cup \overline{58}$ if $i=1,6,7$ and $\mu \cup \mu_{i}^{\prime}$ if $i=2,3,4,5$. Assume that $\operatorname{lk}\left(f\left(\mu_{i}^{\prime}\right)\right)$ is odd for some $i$. Then $J^{i}$ contains a graph $D^{i}$ as a minor such that $D^{i}$ is isomorphic to $D_{4}$ and $\left\{\mu, \mu_{i}^{\prime}\right\}=$ $\Psi_{D^{i}, J^{i}}^{(2)}\left(\Gamma^{(2)}\left(D^{i}\right)\right)$. Because both $\operatorname{lk}(f(\mu))$ and $\operatorname{lk}\left(f\left(\mu_{i}^{\prime}\right)\right)$ are odd, by Lemma 4.3 there exists an element $\gamma$ in $\Gamma\left(J^{i}\right)$ such that $a_{2}(f(\gamma))$ is odd.

Case 2. Let $\mu=\left[\begin{array}{lll}24 & 5 & 8\end{array}\right] \cup\left[\begin{array}{llll}1 & 10 & 396\end{array}\right]$. Note that $N_{10}^{\prime}$ contains another $P_{9}$ as the proper minor

$$
\left.\left(\left(\left(\left(N_{10}^{\prime}-\overline{82}\right)-\overline{85}\right)-\overline{87}\right)-\overline{89}\right)-\overline{810}\right)-\overline{34} .
$$

Thus by Lemma 4.1 , there is an element $v^{\prime}$ in $\Gamma^{(2)}\left(P_{9}\right)$ such that $\operatorname{lk}\left(\psi_{N_{10}^{\prime}, P_{9}}(f)\left(v^{\prime}\right)\right)$ is odd. Hence by Proposition 2.1, there exists an element $\mu^{\prime}$ in $\Psi_{P_{9}, N_{10}^{\prime}}^{(2)}\left(\Gamma^{(2)}\left(P_{9}\right)\right)$ such that $\operatorname{lk}\left(f\left(\mu^{\prime}\right)\right)$ is odd. $\Psi_{P_{9}, N_{10}^{\prime}}^{(2)}\left(\Gamma^{(2)}\left(P_{9}\right)\right)$ consists of the seven elements

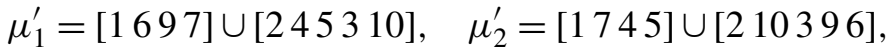

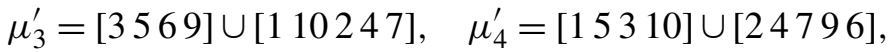

$$
\begin{aligned}
& \mu_{5}^{\prime}=\left[\begin{array}{lll}
1 & 1026
\end{array}\right] \cup\left[\begin{array}{lll}
39745
\end{array}\right], \quad \mu_{6}^{\prime}=[156] \cup[2479310] \text {, }
\end{aligned}
$$

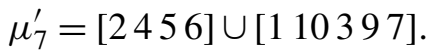

For $i=1,2, \ldots, 7$, let $J^{i}$ be the subgraph of $N_{10}^{\prime}$ that is $\mu \cup \mu_{i}^{\prime} \cup \overline{78}$ if $i=1,7$ and $\mu \cup \mu_{i}^{\prime}$ if $i \neq 1,7$. Assume that $\operatorname{lk}\left(f\left(\mu_{i}^{\prime}\right)\right)$ is odd for some $i$. Then $J^{i}$ contains 
a graph $D^{i}$ as a minor such that $D^{i}$ is isomorphic to $D_{4}$ and

$$
\left\{\mu, \mu_{i}^{\prime}\right\}=\Psi_{D^{i}, J^{i}}^{(2)}\left(\Gamma^{(2)}\left(D^{i}\right)\right) .
$$

Since both $\operatorname{lk}(f(\mu))$ and $\operatorname{lk}\left(f\left(\mu_{i}^{\prime}\right)\right)$ are odd, by Lemma 4.3 there exists an element $\gamma$ in $\Gamma\left(J^{i}\right)$ such that $a_{2}(f(\gamma))$ is odd.

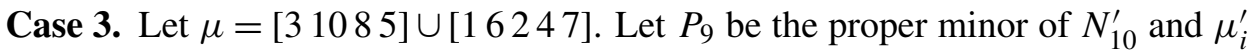
the element in

$$
\Psi_{P_{9}, N_{10}^{\prime}}^{(2)}\left(\Gamma^{(2)}\left(P_{9}\right)\right) \quad(i=1,2, \ldots, 7)
$$

as in Case 2. For $i=1,2, \ldots, 7$, let $J^{i}$ be the subgraph of $N_{10}^{\prime}$ that is $\mu \cup \mu_{i}^{\prime} \cup \overline{89}$ if $i=1,4$ and $\mu \cup \mu_{i}^{\prime}$ if $i \neq 1$, 4. Assume that $\operatorname{lk}\left(f\left(\mu_{i}^{\prime}\right)\right)$ is odd for some $i$. Then $J^{i}$ contains a graph $D^{i}$ as a minor such that $D^{i}$ is isomorphic to $D_{4}$ and $\left\{\mu, \mu_{i}^{\prime}\right\}=\Psi_{D^{i}, J^{i}}^{(2)}\left(\Gamma^{(2)}\left(D^{i}\right)\right)$. Because both $\operatorname{lk}(f(\mu))$ and $\operatorname{lk}\left(f\left(\mu_{i}^{\prime}\right)\right)$ are odd, by Lemma 4.3 there exists an element $\gamma$ in $\Gamma\left(J^{i}\right)$ such that $a_{2}(f(\gamma))$ is odd.

Case 4. Let $\mu=\left[\begin{array}{lll}3 & 45\end{array}\right] \cup\left[\begin{array}{lll}1 & 10 & 26\end{array}\right]$. Note that $N_{10}^{\prime}$ contains another $P_{7}$ as the proper minor

$$
\left(\left(\left(N_{10}^{\prime}-\overline{34}\right)-\overline{45}\right)-\overline{53}\right) / \overline{39} / \overline{47} / \overline{58} .
$$

Thus by Lemma 4.1 , there is an element $v^{\prime}$ in $\Gamma^{(2)}\left(P_{7}\right)$ such that $\operatorname{lk}\left(\psi_{N_{10}^{\prime}, P_{7}}(f)\left(v^{\prime}\right)\right)$ is odd. Hence by Proposition 2.1, there exists an element $\mu^{\prime}$ in $\Psi_{P_{7}, N_{10}^{\prime}}^{(2)}\left(\Gamma^{(2)}\left(P_{7}\right)\right)$ such that $\operatorname{lk}\left(f\left(\mu^{\prime}\right)\right)$ is odd. $\Psi_{P_{7}, N_{10}^{\prime}}^{(2)}\left(\Gamma^{(2)}\left(P_{7}\right)\right)$ consists of the nine elements

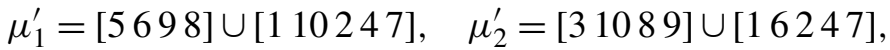

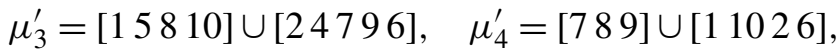

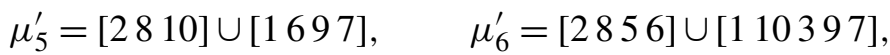

$$
\begin{aligned}
& \mu_{7}^{\prime}=\left[\begin{array}{lll}
1 & 785
\end{array}\right] \cup[210396], \quad \mu_{8}^{\prime}=[156] \cup[2479310] \text {, }
\end{aligned}
$$

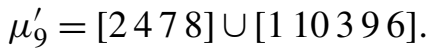

For $i=1,2, \ldots, 9$, let $J^{i}$ be the subgraph of $N_{10}^{\prime}$ that is $\mu \cup \mu_{5}^{\prime} \cup \overline{47} \cup \overline{58}$ if $i=5$ and $\mu \cup \mu_{i}^{\prime}$ if $i \neq 5$. Assume that $\operatorname{lk}\left(f\left(\mu_{i}^{\prime}\right)\right)$ is odd for some $i \neq$ 4,8 . Then $J^{i}$ contains a graph $D^{i}$ as a minor such that $D^{i}$ is isomorphic to $D_{4}$ and $\left\{\mu, \mu_{i}^{\prime}\right\}=\Psi_{D^{i}, J^{i}}^{(2)}\left(\Gamma^{(2)}\left(D^{i}\right)\right)$. Since both $\operatorname{lk}(f(\mu))$ and $\operatorname{lk}\left(f\left(\mu_{i}^{\prime}\right)\right)$ are odd, by Lemma 4.3 there exists an element $\gamma$ in $\Gamma\left(J^{i}\right)$ such that $a_{2}(f(\gamma))$ is odd. Next assume that $\operatorname{lk}\left(f\left(\mu_{8}^{\prime}\right)\right)$ is odd. We denote two elements [1 56] $\cup[24310]$ and [156] $\cup[3479]$ in $\Gamma^{(2)}\left(J^{8}\right)$ by $\mu_{8,1}^{\prime}$ and $\mu_{8,2}^{\prime}$, respectively. We denote the subgraph $\mu \cup \mu_{8,1}^{\prime}$ of $J^{8}$ by $J^{8,1}$ and the subgraph $\mu \cup \mu_{8,2}^{\prime} \cup \overline{89} \cup \overline{810}$ of $N_{10}^{\prime}$ by $J^{8,2}$. Then $J^{8, j}$ contains a graph $D^{8, j}$ as a minor such that $D^{8, j}$ is isomorphic to $D_{4}$ and $\left\{\mu, \mu_{8, j}^{\prime}\right\}=\Psi_{D^{8, j}, J^{8, j}}^{(2)}\left(\Gamma^{(2)}\left(D^{8, j}\right)\right)(j=1,2)$. Since [2 479310$]=$ [24310] $+[3479]$ in $H_{1}\left(J^{8} ; \mathbb{Z}_{2}\right)$, it follows that

$$
1 \equiv \operatorname{lk}\left(f\left(\mu_{8}^{\prime}\right)\right) \equiv \operatorname{lk}\left(f\left(\mu_{8,1}^{\prime}\right)\right)+\operatorname{lk}\left(f\left(\mu_{8,2}^{\prime}\right)\right)(\bmod 2) .
$$


This implies that $\operatorname{lk}\left(f\left(\mu_{8,1}^{\prime}\right)\right)$ is odd or $\operatorname{lk}\left(f\left(\mu_{8,2}^{\prime}\right)\right)$ is odd. In either case, by Lemma 4.3 there exists an element $\gamma$ in $\Gamma\left(J^{8, j}\right)$ such that $a_{2}(f(\gamma))$ is odd. Finally assume that $\operatorname{lk}\left(f\left(\mu_{4}^{\prime}\right)\right)$ is odd. Note that $N_{10}^{\prime}$ contains another $P_{9}$ as the proper minor

$$
\left.\left(\left(\left(\left(N_{10}^{\prime}-\overline{24}\right)-\overline{26}\right)-\overline{28}\right)-\overline{210}\right)-\overline{51}\right)-\overline{53} .
$$

Thus by Lemma 4.1, there is an element $\nu^{\prime \prime}$ in $\Gamma^{(2)}\left(P_{9}\right)$ such that $\operatorname{lk}\left(\psi_{N_{10}^{\prime}, P_{9}}(f)\left(v^{\prime \prime}\right)\right)$ is odd. Hence by Proposition 2.1, there exists an element $\mu^{\prime \prime}$ in $\Psi_{P_{9}, N_{10}^{\prime}}^{(2)}\left(\Gamma^{(2)}\left(P_{9}\right)\right)$ such that $\operatorname{lk}\left(f\left(\mu^{\prime \prime}\right)\right)$ is odd. $\Psi_{P_{9}, N_{10}^{\prime}}^{(2)}\left(\Gamma^{(2)}\left(P_{9}\right)\right)$ consists of the seven elements

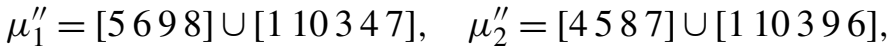

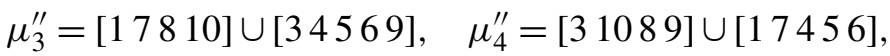

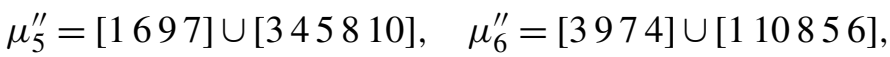

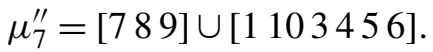

For $j=1,2, \ldots, 7$, let $J^{4, j}$ be the subgraph of $N_{10}^{\prime}$ which is $\mu_{4}^{\prime} \cup \mu_{j}^{\prime \prime} \cup \overline{24}$ if $j=2,6$ and $\mu_{4}^{\prime} \cup \mu_{j}^{\prime \prime}$ if $j \neq 2,6$. Assume that $\operatorname{lk}\left(f\left(\mu_{j}^{\prime \prime}\right)\right)$ is odd for some $j \neq 7$. Then $J^{4, j}$ contains a graph $D^{4, j}$ as a minor such that $D^{4, j}$ is isomorphic to $D_{4}$ and $\left\{\mu_{4}^{\prime}, \mu_{i}^{\prime \prime}\right\}=\Psi_{D^{4, j}, J^{4, j}}^{(2)}\left(\Gamma^{(2)}\left(D^{4, j}\right)\right)$. Since both $\operatorname{lk}\left(f\left(\mu_{4}^{\prime}\right)\right)$ and $\operatorname{lk}\left(f\left(\mu_{j}^{\prime \prime}\right)\right)$ are odd, by Lemma 4.3 there exists an element $\gamma$ in $\Gamma\left(J^{4, j}\right)$ such that $a_{2}(f(\gamma))$ is odd. Next assume that $\operatorname{lk}\left(f\left(\mu_{7}^{\prime \prime}\right)\right)$ is odd. We denote three elements [7 89 9] $\cup$ [1 53 10],

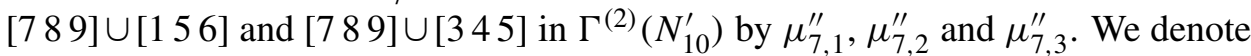
the subgraph $\mu \cup \mu_{7, k}^{\prime \prime} \cup \overline{47} \cup \overline{28}$ of $N_{10}^{\prime}$ by $J^{4,7, k}(k=1,2)$. Then $J^{4,7, k}$ contains a graph $D^{4,7, k}$ as a minor such that $D^{4,7, k}$ is isomorphic to $D_{4}$ and $\left\{\mu, \mu_{7, k}^{\prime \prime}\right\}=$ $\Psi_{D^{4,7, k}, J^{4,7, k}}^{(2)}\left(\Gamma^{(2)}\left(D^{4,7, k}\right)\right)(k=1,2)$. Since [1 103456] $=\left[\begin{array}{ll}15310 & 103\end{array}+[156]+\right.$ [3 4 5] in $H_{1}\left(N_{10}^{\prime} ; \mathbb{Z}_{2}\right)$, it follows that

$$
1 \equiv \operatorname{lk}\left(f\left(\mu_{7}^{\prime}\right)\right) \equiv \operatorname{lk}\left(f\left(\mu_{7,1}^{\prime \prime}\right)\right)+\operatorname{lk}\left(f\left(\mu_{7,2}^{\prime \prime}\right)\right)+\operatorname{lk}\left(f\left(\mu_{7,3}^{\prime \prime}\right)\right)(\bmod 2) .
$$

This implies that $\operatorname{lk}\left(f\left(\mu_{7, k}^{\prime \prime}\right)\right)$ is odd for some $k$. If $\operatorname{lk}\left(f\left(\mu_{7,1}^{\prime \prime}\right)\right)$ is odd or $\operatorname{lk}\left(f\left(\mu_{7,2}^{\prime \prime}\right)\right)$ is odd, then by Lemma 4.3 there exists an element $\gamma$ in $\Gamma\left(J^{4,7, k}\right)$ such that $a_{2}(f(\gamma))$ is odd. If $\operatorname{lk}\left(f\left(\mu_{7,3}^{\prime \prime}\right)\right)$ is odd, let us consider the 3-component link

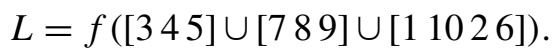

Since all 2-component sublinks of $L$ are $f(\mu), f\left(\mu_{4}^{\prime}\right)$ and $f\left(\mu_{7,3}^{\prime \prime}\right)$, each of the 2-component sublinks of $L$ has an odd linking number.

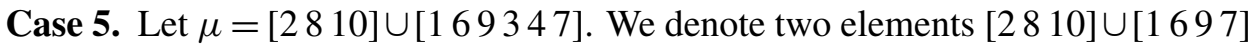
and [2 810$] \cup[3974]$ in $\Gamma^{(2)}\left(N_{10}^{\prime}\right)$ by $\mu_{1}$ and $\mu_{2}$, respectively. Since [1 69347$]=$ [1697] $+[3974]$ in $H_{1}\left(N_{10}^{\prime} ; \mathbb{Z}_{2}\right)$, it follows that

$$
1 \equiv \mathrm{lk}(f(\mu)) \equiv \operatorname{lk}\left(f\left(\mu_{1}\right)\right)+\operatorname{lk}\left(f\left(\mu_{2}\right)\right)(\bmod 2) .
$$


This implies that $\operatorname{lk}\left(f\left(\mu_{1}\right)\right)$ is odd or $\operatorname{lk}\left(f\left(\mu_{2}\right)\right)$ is odd. By the symmetry of $N_{10}^{\prime}$, we may assume that $\operatorname{lk}\left(f\left(\mu_{1}\right)\right)$ is odd. Note that $N_{10}^{\prime}$ contains another $P_{7}$ as the proper minor

$$
\left(\left(\left(N_{10}^{\prime}-\overline{28}\right)-\overline{810}\right)-\overline{102}\right) / \overline{26} / \overline{310} / \overline{58} .
$$

Thus by Lemma 4.1, there is an element $v^{\prime}$ in $\Gamma^{(2)}\left(P_{7}\right)$ such that $\operatorname{lk}\left(\psi_{N_{10}^{\prime}, P_{7}}(f)\left(v^{\prime}\right)\right)$ is odd. Hence by Proposition 2.1, there exists an element $\mu^{\prime}$ in $\Psi_{P_{7}, N_{10}^{\prime}}^{(2)}\left(\Gamma^{(2)}\left(P_{7}\right)\right)$ such that $\operatorname{lk}\left(f\left(\mu^{\prime}\right)\right)$ is odd. $\Psi_{P_{7}, N_{10}^{\prime}}^{(2)}\left(\Gamma^{(2)}\left(P_{7}\right)\right)$ consists of the nine elements

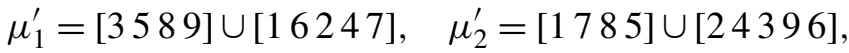

$$
\begin{aligned}
& \mu_{3}^{\prime}=[156] \cup[3974], \quad \mu_{4}^{\prime}=[345] \cup[1697] \text {, }
\end{aligned}
$$

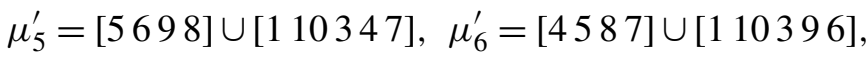

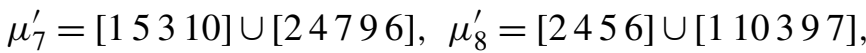

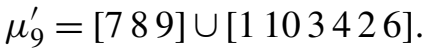

For $i=1,2, \ldots, 9$, let $J^{i}$ be the subgraph of $N_{10}^{\prime}$ that is $\mu_{1} \cup \mu_{3}^{\prime} \cup \overline{310} \cup \overline{58}$ if $i=3$ and $\mu_{1} \cup \mu_{i}^{\prime}$ if $i \neq 3$. Assume that $\operatorname{lk}\left(f\left(\mu_{i}^{\prime}\right)\right)$ is odd for some $i \neq 4,9$. Then $J^{i}$ contains a graph $D^{i}$ as a minor such that $D^{i}$ is isomorphic to $D_{4}$ and $\left\{\mu_{1}, \mu_{i}^{\prime}\right\}=\Psi_{D^{i}, J^{i}}^{(2)}\left(\Gamma^{(2)}\left(D^{i}\right)\right)$. Since both $\operatorname{lk}\left(f\left(\mu_{1}\right)\right)$ and $\operatorname{lk}\left(f\left(\mu_{i}^{\prime}\right)\right)$ are odd, by Lemma 4.3 there exists an element $\gamma$ in $\Gamma\left(J^{i}\right)$ such that $a_{2}(f(\gamma))$ is odd. Next assume that $\operatorname{lk}\left(f\left(\mu_{9}^{\prime}\right)\right)$ is odd. We denote two elements [7 89] $\cup$ [1 62 10] and [7 8 9] $\cup[24310]$ in $\Gamma^{(2)}\left(J^{9}\right)$ by $\mu_{9,1}^{\prime}$ and $\mu_{9,2}^{\prime}$, respectively. We denote the subgraph $\mu_{1} \cup \mu_{8,1}^{\prime}$ of $J^{9}$ by $J^{9,1}$ and the subgraph $\mu_{1} \cup \mu_{9,2}^{\prime} \cup \overline{53} \cup \overline{51}$ of $N_{10}^{\prime}$ by $J^{9,2}$. Then $J^{9, j}$ contains a graph $D^{9, j}$ as a minor such that $D^{9, j}$ is isomorphic to $D_{4}$ and

$$
\left\{\mu_{1}, \mu_{9, j}^{\prime}\right\}=\Psi_{D^{9, j}, J^{9, j}}^{(2)}\left(\Gamma^{(2)}\left(D^{9, j}\right)\right) \quad(j=1,2) .
$$

Since $\left[\begin{array}{lllll}1 & 103 & 4 & 26\end{array}\right]=\left[\begin{array}{lll}1 & 62 & 10\end{array}\right]+[24310]$ in $H_{1}\left(J^{9} ; \mathbb{Z}_{2}\right)$, it follows that

$$
1 \equiv \mathrm{lk}\left(f\left(\mu_{9}^{\prime}\right)\right) \equiv \mathrm{lk}\left(f\left(\mu_{9,1}^{\prime}\right)\right)+\mathrm{lk}\left(f\left(\mu_{9,2}^{\prime}\right)\right)(\bmod 2)
$$

This implies that $\operatorname{lk}\left(f\left(\mu_{9,1}^{\prime}\right)\right)$ is odd or $\operatorname{lk}\left(f\left(\mu_{9,2}^{\prime}\right)\right)$ is odd. In either case, by Lemma 4.3 there exists an element $\gamma$ in $\Gamma\left(J^{9, j}\right)$ such that $a_{2}(f(\gamma))$ is odd. Finally assume that $\operatorname{lk}\left(f\left(\mu_{4}^{\prime}\right)\right)$ is odd. $N_{10}^{\prime}$ contains another $P_{9}$ as the proper minor

$$
\left.\left(\left(\left(\left(N_{10}^{\prime}-\overline{61}\right)-\overline{62}\right)-\overline{65}\right)-\overline{69}\right)-\overline{87}\right)-\overline{810} .
$$

Thus, by Lemma 4.1, there is $v^{\prime \prime} \in \Gamma^{(2)}\left(P_{9}\right)$ such that $\operatorname{lk}\left(\psi_{N_{10}^{\prime}, P_{9}}(f)\left(v^{\prime \prime}\right)\right)$ is odd. Hence by Proposition 2.1, there exists $\mu^{\prime \prime} \in \Psi_{P_{9}, N_{10}^{\prime}}^{(2)}\left(\Gamma^{(2)}\left(P_{9}\right)\right)$ such that $\operatorname{lk}\left(f\left(\mu^{\prime \prime}\right)\right)$ 
is odd. The set $\Psi_{P_{9}, N_{10}^{\prime}}^{(2)}\left(\Gamma^{(2)}\left(P_{9}\right)\right)$ consists of the seven elements

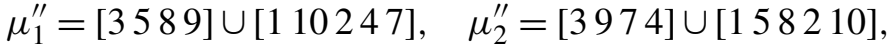

$$
\begin{aligned}
& \mu_{3}^{\prime \prime}=[1745] \cup[289310], \quad \mu_{4}^{\prime \prime}=[2458] \cup[110397] \text {, }
\end{aligned}
$$

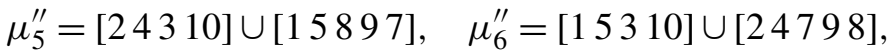

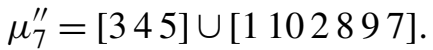

For $j=1,2, \ldots, 7$, let $J^{4, j}$ be the subgraph of $N_{10}^{\prime}$ that is $\mu_{4}^{\prime} \cup \mu_{j}^{\prime \prime} \cup \overline{26}$ if $j=4,5$ and $\mu_{4}^{\prime} \cup \mu_{j}^{\prime \prime}$ if $j \neq 4,5$. Assume that $\operatorname{lk}\left(f\left(\mu_{j}^{\prime \prime}\right)\right)$ is odd for some $j \neq 7$. Then $J^{4, j}$ contains a graph $D^{4, j}$ as a minor such that $D^{4, j}$ is isomorphic to $D_{4}$ and $\left\{\mu_{4}^{\prime}, \mu_{i}^{\prime \prime}\right\}=\Psi_{D^{4, j}, J^{4, j}}^{(2)}\left(\Gamma^{(2)}\left(D^{4, j}\right)\right)$. Since both $\operatorname{lk}\left(f\left(\mu_{4}^{\prime}\right)\right)$ and $\operatorname{lk}\left(f\left(\mu_{j}^{\prime \prime}\right)\right)$ are odd, by Lemma 4.3 there exists an element $\gamma$ in $\Gamma\left(J^{4, j}\right)$ such that $a_{2}(f(\gamma))$ is odd.

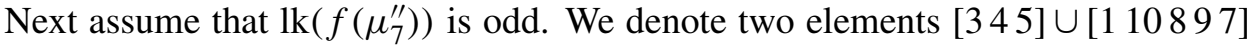
and [3 45] $\cup[2810]$ in $\Gamma^{(2)}\left(N_{10}^{\prime}\right)$ by $\mu_{7,1}^{\prime \prime}$ and $\mu_{7,2}^{\prime \prime}$, respectively. We denote the subgraph $\mu_{1} \cup \mu_{7,1}^{\prime \prime} \cup \overline{24} \cup \overline{56}$ of $N_{10}^{\prime}$ by $J^{4,7}$. Then $J^{4,7}$ contains a graph $D^{4,7}$ as a minor such that $D^{4,7}$ is isomorphic to $D_{4}$ and

$$
\left\{\mu_{1}, \mu_{7,1}^{\prime \prime}\right\}=\Psi_{D^{4,7}, J^{4,7}}^{(2)}\left(\Gamma^{(2)}\left(D^{4,7}\right)\right) .
$$

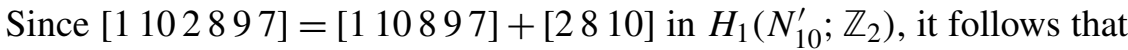

$$
1 \equiv \operatorname{lk}\left(f\left(\mu_{7}^{\prime}\right)\right) \equiv \operatorname{lk}\left(f\left(\mu_{7,1}^{\prime \prime}\right)\right)+\operatorname{lk}\left(f\left(\mu_{7,2}^{\prime \prime}\right)\right)(\bmod 2) .
$$

This implies that $\operatorname{lk}\left(f\left(\mu_{7,1}^{\prime \prime}\right)\right)$ is odd or $\operatorname{lk}\left(f\left(\mu_{7,2}^{\prime \prime}\right)\right)$ is odd. If $\operatorname{lk}\left(f\left(\mu_{7,1}^{\prime \prime}\right)\right)$ is odd, then by Lemma 4.3 there exists an element $\gamma$ in $\Gamma\left(J^{4,7}\right)$ such that $a_{2}(f(\gamma))$ is odd. If $\operatorname{lk}\left(f\left(\mu_{7,2}^{\prime \prime}\right)\right)$ is odd, let us consider the 3-component link

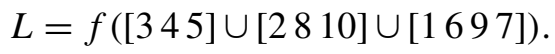

Since all 2-component sublinks of $L$ are $f\left(\mu_{1}\right), f\left(\mu_{4}^{\prime}\right)$ and $f\left(\mu_{7,2}^{\prime \prime}\right)$, each of the 2component sublinks of $L$ has an odd linking number. This completes the proof.

Proof of Theorem 1.2. A graph in the Heawood family is obtained from one of $K_{7}, N_{9}$ and $N_{10}^{\prime}$ by a finite sequence of $\triangle \mathrm{Y}$-exchanges. Thus by Lemma 2.6, Theorem 4.4, and the fact that $K_{7}$ is $\mathrm{IK}$ - and thus $\mathrm{I}(\mathrm{K}$ or $\mathrm{C} 3 \mathrm{~L})$ - it follows that every graph in the Heawood family is $\mathrm{I}(\mathrm{K}$ or $\mathrm{C} 3 \mathrm{~L})$. On the other hand, a graph in the Heawood family is obtained from one of $H_{12}$ and $C_{14}$ by a finite sequence of Y $\triangle$-exchanges. Since each of $H_{12}$ and $C_{14}$ is a minor-minimal IK graph and $\Gamma^{(3)}\left(H_{12}\right)$ and $\Gamma^{(3)}\left(C_{14}\right)$ are the empty sets, it follows that $H_{12}$ and $C_{14}$ are minorminimal $\mathrm{I}(\mathrm{K}$ or $\mathrm{C} 3 \mathrm{~L})$ graphs. By Lemma 2.7, we have the desired conclusion.

Remark 4.5. A graph is said to be 2-apex if it can be embedded in the 2-sphere after the deletion of at most two vertices and all of their incidental edges. It is not hard to see that any 2-apex graph may have a spatial embedding whose image 
contains neither a nontrivial knot nor a 3-component link all of whose 2-component sublinks are nonsplittable. Thus any 2-apex graph is not $\mathrm{I}(\mathrm{K}$ or $\mathrm{C} 3 \mathrm{~L})$. It is known that every graph of at most twenty edges is 2-apex [Mattman 2011] (see also [Johnson et al. 2010]). Since the number of all edges of every graph in the Heawood family is twenty-one, we see that any proper minor of a graph in the Heawood family is 2-apex, and thus not $\mathrm{I}(\mathrm{K}$ or $\mathrm{C} 3 \mathrm{~L})$. This also implies that any graph in the Heawood family is minor-minimal for $\mathrm{I}(\mathrm{K}$ or $\mathrm{C} 3 \mathrm{~L})$.

Example 4.6. Let $g_{9}$ be the spatial embedding of $N_{9}$ and $g_{10}^{\prime}$ the spatial embedding of $N_{10}^{\prime}$ illustrated here:

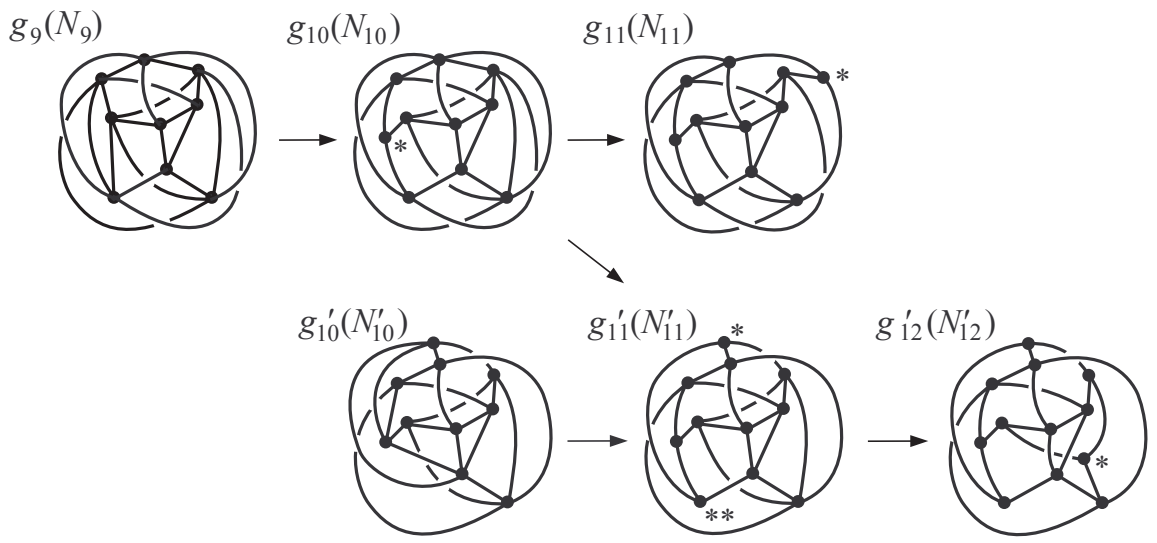

Then it can be checked directly that both $g_{9}\left(N_{9}\right)$ and $g_{10}^{\prime}\left(N_{10}^{\prime}\right)$ do not contain a nonsplittable 3-component link. Thus neither $N_{9}$ nor $N_{10}^{\prime}$ is I3L. Also, we can see that $N_{10}, N_{11}, N_{11}^{\prime}$ and $N_{12}^{\prime}$ are not I3L in a similar way as the proof of Lemma 3.1 (see figure above).

Remark 4.7. The Heawood graph is IK. The Heawood graph $H$ is the dual graph of $K_{7}$, which is embedded in a torus. It is known that there exists a unique graph $C_{14}$ obtained from $K_{7}$ by seven applications of $\triangle \mathrm{Y}$-exchanges [Kohara and Suzuki 1992]. The seven triangles correspond to the black triangles of a black-and-white coloring of the torus by $K_{7}$. Then $C_{14}$ and $H$ are mapped to each other by a translation of the torus:
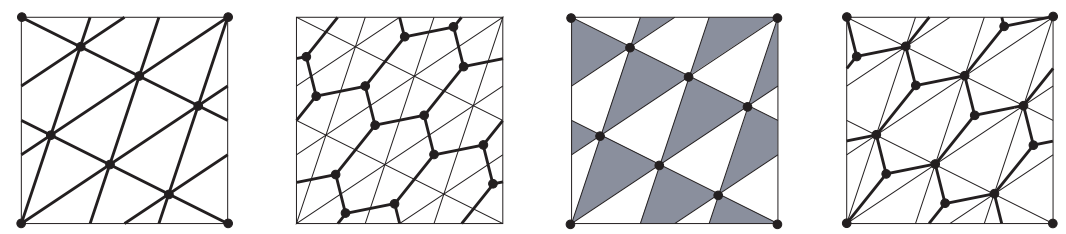

Thus they are isomorphic. Since $C_{14}$ is IK, we have the result. 
Remark 4.8. It is known that all twenty-six graphs obtained from the complete four-partite graph $K_{3,3,1,1}$ by a finite sequence of $\triangle \mathrm{Y}$-exchanges are minor-minimal IK graphs [Kohara and Suzuki 1992; Foisy 2002]. There exist thirty-two graphs that are obtained from $K_{3,3,1,1}$ by a finite sequence of $\triangle \mathrm{Y}$-exchanges and $\mathrm{Y} \triangle$ exchanges but that cannot be obtained from $K_{3,3,1,1}$ by a finite sequence of $\Delta \mathrm{Y}$ exchanges. Recently, Goldberg, Mattman, and Naimi [2011] announced that these thirty-two graphs are also minor-minimal IK graphs.

\section{References}

[Conway and Gordon 1983] J. H. Conway and C. M. Gordon, "Knots and links in spatial graphs", J. Graph Theory 7:4 (1983), 445-453. MR 85d:57002 Zbl 0524.05028

[Fellows and Langston 1988] M. R. Fellows and M. A. Langston, "Nonconstructive tools for proving polynomial-time decidability", J. Assoc. Comput. Mach. 35:3 (1988), 727-739. MR 90i:68046 Zbl 0652.68049

[Flapan and Naimi 2008] E. Flapan and R. Naimi, "The Y-triangle move does not preserve intrinsic knottedness", Osaka J. Math. 45:1 (2008), 107-111. MR 2009b:05078 Zbl 1145.05019

[Flapan et al. 2001a] E. Flapan, R. Naimi, and J. Pommersheim, "Intrinsically triple linked complete graphs”, Topology Appl. 115:2 (2001), 239-246. MR 2002f:57007 Zbl 0988.57003

[Flapan et al. 2001b] E. Flapan, J. Pommersheim, J. Foisy, and R. Naimi, "Intrinsically $n$-linked graphs”, J. Knot Theory Ramifications 10:8 (2001), 1143-1154. MR 2003a:57002 Zb1 0998.57008

[Foisy 2002] J. Foisy, "Intrinsically knotted graphs", J. Graph Theory 39:3 (2002), 178-187. MR 2003a:05051 Zbl 1176.05022

[Foisy 2003] J. Foisy, "A newly recognized intrinsically knotted graph", J. Graph Theory 43:3 (2003), 199-209. MR 2004c:05058 Zbl 1022.05019

[Foisy 2006] J. Foisy, "Graphs with a knot or 3-component link in every spatial embedding", J. Knot Theory Ramifications 15:9 (2006), 1113-1118. MR 2008a:05068 Zbl 1119.57001

[Goldberg et al. 2011] N. Goldberg, T. Mattman, and R. Naimi, "Many, many more minor minimal intrinsically knotted graphs", preprint, 2011. arXiv 1109.1632

[van der Holst 2006] H. van der Holst, "Graphs and obstructions in four dimensions", J. Combin. Theory Ser. B 96:3 (2006), 388-404. MR 2007a:05041 Zbl 1088.05067

[Johnson et al. 2010] B. Johnson, M. E. Kidwell, and T. S. Michael, "Intrinsically knotted graphs have at least 21 edges", J. Knot Theory Ramifications 19:11 (2010), 1423-1429. MR 2746195 Zbl 05835915

[Kauffman 1983] L. H. Kauffman, Formal knot theory, Mathematical Notes 30, Princeton University Press, 1983. MR 85b:57006 Zbl 0537.57002

[Kohara and Suzuki 1992] T. Kohara and S. Suzuki, "Some remarks on knots and links in spatial graphs”, pp. 435-445 in Knots 90 (Osaka, 1990), edited by A. Kawauchi, de Gruyter, Berlin, 1992. MR 93i:57004 Zbl 0771.57002

[Mattman 2011] T. W. Mattman, "Graphs of 20 edges are 2-apex, hence unknotted", Algebr. Geom. Topol. 11:2 (2011), 691-718. MR 2011m:05106 Zbl 1216.05017

[Motwani et al. 1988] R. Motwani, A. Raghunathan, and H. Saran, "Constructive results from graph minors: Linkless embeddings", pp. 398-409 in 29th Annual Symposium on Foundations of Computer Science (White Plains, NY, 1988), 1988. 
[Nešetřil and Thomas 1985] J. Nešetřil and R. Thomas, "A note on spatial representation of graphs", Comment. Math. Univ. Carolin. 26:4 (1985), 655-659. MR 87e:05063 Zbl 0602.05024

[Ozawa and Tsutsumi 2007] M. Ozawa and Y. Tsutsumi, "Primitive spatial graphs and graph minors", Rev. Mat. Complut. 20:2 (2007), 391-406. MR 2008g:57005 Zbl 1142.57004

[Robertson and Seymour 2004] N. Robertson and P. D. Seymour, "Graph minors, XX: Wagner's conjecture", J. Combin. Theory Ser. B 92:2 (2004), 325-357. MR 2005m:05204 Zbl 1061.05088

[Robertson et al. 1995] N. Robertson, P. Seymour, and R. Thomas, "Sachs' linkless embedding conjecture”, J. Combin. Theory Ser. B 64:2 (1995), 185-227. MR 96m:05072 Zbl 0832.05032

[Sachs 1984] H. Sachs, "On spatial representations of finite graphs", pp. 649-662 in Finite and infinite sets (Eger, 1981), vol. 2, edited by A. Hajnal et al., Colloq. Math. Soc. János Bolyai 37, North-Holland, Amsterdam, 1984. MR 87c:05055 Zbl 0568.05026

[Taniyama and Yasuhara 2001] K. Taniyama and A. Yasuhara, "Realization of knots and links in a spatial graph”, Topology Appl. 112:1 (2001), 87-109. MR 2002e:57005 Zbl 0968.57001

Received August 10, 2010. Revised January 20, 2011.

RYO HANAKI

DEPARTMENT OF MATHEMATICS

NARA UNIVERSITY OF EDUCATION

TAKABATAKE

NARA 630-8305

JAPAN

hanaki@nara-edu.ac.jp

RYO NIKKUNI

Department of MATHEMATICS, SCHOOL OF ARTS AND SCIENCES

TOKYO WOMAN'S CHRISTIAN UNIVERSITY

2-6-1 ZEMPUKUJI, SUGINAMI-KU

TOKYO 167-8585

JAPAN

nick@lab.twcu.ac.jp

KOUKI TANIYAMA

DePartment of Mathematics, School of Education

WASEDA UNIVERSITY

NISHI-WASEDA 1-6-1, SHINJUKU-KU

TOKYO 169-8050

JAPAN

taniyama@waseda.jp

AKIKO YAMAZAKI

Division of Mathematics, GRaduate SCHOOL of SCIENCE

TOKYO WOMAN'S CHRISTIAN UNIVERSITY

2-6-1 ZEMPUKUJI, SUGINAMI-KU

TOKYO 167-8585

JAPAN

smilebimoch@khc.biglobe.ne.jp 


\title{
PACIFIC JOURNAL OF MATHEMATICS
}

\author{
http://www.pjmath.org \\ Founded in 1951 by
}

E. F. Beckenbach (1906-1982) and F. Wolf (1904-1989)

\section{EDITORS}

V. S. Varadarajan (Managing Editor)

Department of Mathematics

University of California

Los Angeles, CA 90095-1555

pacific@math.ucla.edu

Vyjayanthi Chari

Department of Mathematics

University of California

Riverside, CA 92521-0135

chari@math.ucr.edu

\section{Robert Finn}

Department of Mathematics Stanford University

Stanford, CA 94305-2125

finn@math.stanford.edu

Kefeng Liu

Department of Mathematics

University of California

Los Angeles, CA 90095-1555

liu@math.ucla.edu
Darren Long

Department of Mathematics

University of California

Santa Barbara, CA 93106-3080

long@math.ucsb.edu

Jiang-Hua Lu

Department of Mathematics

The University of Hong Kong

Pokfulam Rd., Hong Kong jhlu@maths.hku.hk

Alexander Merkurjev

Department of Mathematics University of California

Los Angeles, CA 90095-1555 merkurev@math.ucla.edu
Sorin Popa

Department of Mathematics

University of California

Los Angeles, CA 90095-1555

popa@math.ucla.edu

Jie Qing

Department of Mathematics

University of California

Santa Cruz, CA 95064

qing@ cats.ucsc.edu

Jonathan Rogawski

Department of Mathematics

University of California

Los Angeles, CA 90095-1555

jonr@math.ucla.edu

\section{PRODUCTION}

pacific@math.berkeley.edu

Silvio Levy, Scientific Editor Matthew Cargo, Senior Production Editor

ACADEMIA SINICA, TAIPEI

CALIFORNIA INST. OF TECHNOLOGY

INST. DE MATEMÁTICA PURA E APLICADA

KEIO UNIVERSITY

MATH. SCIENCES RESEARCH INSTITUTE

NEW MEXICO STATE UNIV.

OREGON STATE UNIV.

\section{SUPPORTING INSTITUTIONS}

STANFORD UNIVERSITY
UNIV. OF BRITISH COLUMBIA
UNIV. OF CALIFORNIA, BERKELEY
UNIV. OF CALIFORNIA, DAVIS
UNIV. OF CALIFORNIA, LOS ANGELES
UNIV. OF CALIFORNIA, RIVERSIDE
UNIV. OF CALIFORNIA, SAN DIEGO
UNIV. OF CALIF., SANTA BARBARA

UNIV. OF CALIF., SANTA CRUZ

UNIV. OF MONTANA

UNIV. OF OREGON

UNIV. OF SOUTHERN CALIFORNIA

UNIV. OF UTAH

UNIV. OF WASHINGTON

WASHINGTON STATE UNIVERSITY

These supporting institutions contribute to the cost of publication of this Journal, but they are not owners or publishers and have no responsibility for its contents or policies.

See inside back cover or www.pjmath.org for submission instructions.

The subscription price for 2011 is US \$420/year for the electronic version, and \$485/year for print and electronic.

Subscriptions, requests for back issues from the last three years and changes of subscribers address should be sent to Pacific Journal of Mathematics, P.O. Box 4163, Berkeley, CA 94704-0163, U.S.A. Prior back issues are obtainable from Periodicals Service Company, 11 Main Street, Germantown, NY 12526-5635. The Pacific Journal of Mathematics is indexed by Mathematical Reviews, Zentralblatt MATH, PASCAL CNRS Index, Referativnyi Zhurnal, Current Mathematical Publications and the Science Citation Index.

The Pacific Journal of Mathematics (ISSN 0030-8730) at the University of California, c/o Department of Mathematics, 969 Evans Hall, Berkeley, CA 94720-3840, is published monthly except July and August. Periodical rate postage paid at Berkeley, CA 94704, and additional mailing offices. POSTMASTER: send address changes to Pacific Journal of Mathematics, P.O. Box 4163, Berkeley, CA 94704-0163.

PJM peer review and production are managed by EditFLOW ${ }^{\mathrm{TM}}$ from Mathematical Sciences Publishers.

PUBLISHED BY PACIFIC JOURNAL OF MATHEMATICS

at the University of California, Berkeley 94720-3840

A NON-PROFIT CORPORATION

Typeset in IATEX

Copyright $(2011$ by Pacific Journal of Mathematics 


\title{
PACIFIC JOURNAL OF MATHEMATICS
}

\author{
Volume $252 \quad$ No. $2 \quad$ August 2011
}

Remarks on a Künneth formula for foliated de Rham cohomology

MÉLANIE BERTELSON

$K$-groups of the quantum homogeneous space ${ }_{q}(n) /_{q}(n-2)$

275

PARTHA SARATHI CHAKRABORTY and S. SUNDAR

A class of irreducible integrable modules for the extended baby TKK algebra

293

Xuewu Chang and ShaObin TAN

Duality properties for quantum groups

SOPHIE CHEMLA

Representations of the category of modules over pointed Hopf algebras over $\mathbb{S}_{3}$ and $\mathrm{S}_{4}$

Agustín García IGLESIAS and Martín Mombelli

$(p, p)$-Galois representations attached to automorphic forms on ${ }_{n}$

EKNATH GHATE and NARASIMHA KUMAR

On intrinsically knotted or completely 3-linked graphs 407

Ryo Hanaki, Ryo NikKUni, Kouki TaNiYama and AKIKo YAMAZAKI

Connection relations and expansions

Mourad E. H. ISMAIL and Mizan Rahman

Characterizing almost Prüfer $v$-multiplication domains in pullbacks

QING LI

Whitney umbrellas and swallowtails

TAKASHI NISHIMURA

The Koszul property as a topological invariant and measure of singularities

HAL SADOFSKY and BRAD SHELTON

A completely positive map associated with a positive map

ERLING STøRMER

Classification of embedded projective manifolds swept out by rational homogeneous varieties of codimension one

KiWAMU Watanabe

Note on the relations in the tautological ring of $\mu_{g}$ 